\title{
MODULI SPACES AND THE INVERSE GALOIS PROBLEM FOR CUBIC SURFACES
}

\author{
ANDREAS-STEPHAN ELSENHANS AND JÖRG JAHNEL
}

\begin{abstract}
We study the moduli space $\widetilde{\mathscr{M}}$ of marked cubic surfaces. By classical work of A. B. Coble, this has a compactification $\widetilde{M}$, which is linearly acted upon by the group $W\left(E_{6}\right) . \widetilde{M}$ is given as the intersection of 30 cubics in $\mathbf{P}^{9}$. For the morphism $\widetilde{\mathscr{M}} \rightarrow \mathbf{P}(1,2,3,4,5)$ forgetting the marking, followed by Clebsch's invariant map, we give explicit formulas, i.e., Clebsch's invariants are expressed in terms of Coble's irrational invariants. As an application, we give an affirmative answer to the inverse Galois problem for cubic surfaces over $\mathbb{Q}$.
\end{abstract}

\section{INTRODUCTION}

Cubic surfaces have been intensively studied by the geometers of the 19th century. For example, it was proven at that time that there are exactly 27 lines on every smooth cubic surface. Further, the configuration of the 27 lines is highly symmetric. The group of all permutations respecting the intersection pairing is isomorphic to the Weyl group $W\left(E_{6}\right)$ of order 51840 .

The concept of a moduli scheme is by far more recent. Nevertheless, there are two kinds of moduli schemes for smooth cubic surfaces and both have their origins in classical invariant theory.

On one hand, there is the coarse moduli scheme $\mathscr{M}$ of smooth cubic surfaces. This scheme is essentially due to G. Salmon 31] and A. Clebsch [4. In fact, in modern language, Clebsch's result from 1861 states that there is an open embedding $\mathrm{Cl}: \mathscr{M} \hookrightarrow \mathbf{P}(1,2,3,4,5)$ into the weighted projective space of weights $1, \ldots, 5$.

On the other hand, one has the fine moduli scheme $\widetilde{\mathscr{M}}$ of smooth cubic surfaces with a marking on the 27 lines. The marking plays the role of a rigidification and excludes all automorphisms. That is why a fine moduli scheme may exist. It has its origins in the work of A. Cayley [3]. An embedding into $\mathbf{P}^{9}$ as an intersection of 30 cubics is due to A. B. Coble [6] and dates back to the year 1917 .

The two moduli spaces are connected by the canonical, i.e. forgetful, morphism pr: $\widetilde{\mathscr{M}} \rightarrow \mathscr{M}$. This is a finite flat morphism of degree 51840 . Its ramification locus corresponds exactly to the cubic surfaces having nontrivial automorphisms.

Explicit formulas. In Theorem [3.9, we will give an explicit description of $\operatorname{pr}: \widetilde{\mathscr{M}} \rightarrow \mathscr{M}$. In other words, given a smooth cubic surface $C$ with a marking on its 27 lines, we provide explicit formulas expressing Clebsch's invariants of $C$ in

Received by the editors March 15, 2013 and, in revised form, August 11, 2013.

2010 Mathematics Subject Classification. Primary 14J15; Secondary 14J20, 14J26, 14G25.

The first author was supported in part by the Deutsche Forschungsgemeinschaft (DFG) through a funded research project. 
terms of Coble's, so-called irrational, invariants. From a formal point of view, this result seems to be new.

But there can be no doubt that its essence, the existence of such formulas, was clear to A. Coble, as well. Due to the lack of computers they could not be worked out at the time, with the exception of the very first. In fact, our approach is a combination of classical invariant theory with modern computer algebra.

A solution to the equation problem. As the main result of this article, we consider the following application of Theorem 3.9. Given an abstract point on the moduli space of marked cubic surfaces, we deliver an algorithm that produces a concrete cubic surface from it.

This algorithm is a combination of the explicit formulas for pr: $\widetilde{\mathscr{M}} \rightarrow \mathscr{M}$ with an algorithmic solution to the so-called equation problem for cubic surfaces, i.e., the problem to determine a concrete cubic surface from a given value of Clebsch's invariant vector. This was seemingly considered hopeless for a long time, but, today, it essentially comes down to the explicit computation of a Galois descent; cf. A.8 and Algorithm A.10.

A further application. When $C$ is a cubic surface over $\mathbb{Q}$, the absolute Galois group $\operatorname{Gal}(\overline{\mathbb{Q}} / \mathbb{Q})$ operates on the 27 lines. This means, after having fixed a marking on the lines, there is a homomorphism $\rho: \operatorname{Gal}(\overline{\mathbb{Q}} / \mathbb{Q}) \rightarrow W\left(E_{6}\right)$. One says that the Galois group $\operatorname{Gal}(\overline{\mathbb{Q}} / \mathbb{Q})$ acts upon the lines of $C$ via $G:=\operatorname{im} \rho \subseteq W\left(E_{6}\right)$. When no marking is chosen, the subgroup $G$ is determined only up to conjugation.

As an application of the considerations on moduli schemes, we obtain the following affirmative answer to the inverse Galois problem for smooth cubic surfaces over $\mathbb{Q}$.

Theorem 0.1. Let $\mathfrak{g}$ be an arbitrary conjugacy class of subgroups of $W\left(E_{6}\right)$. Then there exists a smooth cubic surface $C$ over $\mathbb{Q}$ such that the Galois group acts upon the lines of $C$ via a subgroup $G \subseteq W\left(E_{6}\right)$ belonging to the conjugacy class $\mathfrak{g}$.

The fundamental idea of the proof is as follows. We describe a twist $\widetilde{\mathscr{M}}_{\rho}$ of $\widetilde{\mathscr{M}}$, representing cubic surfaces with a marking that is acted upon by the absolute Galois group via a prescribed homomorphism $\rho: \operatorname{Gal}(\overline{\mathbb{Q}} / \mathbb{Q}) \rightarrow W\left(E_{6}\right)$. The $\mathbb{Q}$-rational points on this scheme correspond to the cubic surfaces of the type sought after.

We do not have the universal family at our disposal, at least not in a sufficiently explicit form. Thus, we calculate Clebsch's invariants of the cubic surface from the projective coordinates of the point found, i.e. from the irrational invariants of the cubic surface. Finally, we recover the surface from Clebsch's invariants.

The list. The complete list of our examples is available on both author's web pages as a file named kub_fl_letzter_teil.txt. The numbering of the conjugacy classes we use is that produced by gap, version 4.4.12. This numbering is reproducible, at least in our version of gap. It coincides with the numbering used in our previous articles.

\section{The Moduli SCHEME of MARKED CUBIC SURFACES}

The purpose of this section is mainly to fix notation and to recall some results that are more or less known. 
Definitions 1.1. i) Let $S$ be any scheme. Then, by a family of cubic surfaces over $S$ or simply a cubic surface over $S$, we mean a flat morphism $p: C \rightarrow S$ such that there exist a rank-4 vector bundle $\mathscr{E}$ on $S$, a nonzero section $c \in \Gamma(\mathscr{O}(3), \mathbf{P}(\mathscr{E}))$, and an isomorphism $\operatorname{div}(c) \stackrel{\cong}{\longrightarrow} C$ of $S$-schemes.

ii) A line on a smooth cubic surface $p: C \rightarrow S$ is a $\mathbf{P}^{1}$-bundle $l \subset C$ over $S$ such that, for every $x \in S$, one has $\operatorname{deg}_{\mathscr{O}(1)} l_{x}=1$.

iii) A family of marked cubic surfaces over a base scheme $S$ or simply a marked cubic surface over $S$ is a cubic surface $p: C \rightarrow S$ together with a sequence $\left(l_{1}, \ldots, l_{6}\right)$ of six mutually disjoint lines. The sequence $\left(l_{1}, \ldots, l_{6}\right)$ itself will be called a marking on $C$.

Remarks 1.2. i) The $\mathbf{P}^{3}$-bundle $\mathbf{P}(\mathscr{E})$ is not part of the structure of a cubic surface over a base scheme. Nevertheless, at least for $p$ smooth, we have $\left.\mathscr{O}(1)\right|_{C}=$ $\left(\Omega_{C / S}^{\wedge 2}\right)^{\vee} \otimes \mathscr{L}$ for some invertible sheaf $\mathscr{L}$ on $S$. Thus, the class of $\left.\mathscr{O}(1)\right|_{C}$ in $\operatorname{Pic}(C) / p^{*} \operatorname{Pic}(S)$ is completely determined by the datum.

ii) A marked cubic surface is automatically smooth, according to our definition. All its 27 lines are defined over $S$. They may be labelled as $l_{1}, \ldots, l_{6}, l_{1}^{\prime}, \ldots, l_{6}^{\prime}$, $l_{12}^{\prime \prime}, l_{13}^{\prime \prime}, \ldots, l_{56}^{\prime \prime} ;$ cf. [20, Theorem V.4.9].

iii) It has been known since the days of A. Cayley that there are exactly 51840 possible markings for a smooth cubic surface with all 27 lines defined over the base. They are acted upon, in a transitive manner, by a group of that order, which is isomorphic to the Weyl group $W\left(E_{6}\right)$ [26, Theorem 23.9].

Convention 1.3. In this article, we will identify $W\left(E_{6}\right)$ with the permutation group acting on the 27 labels $l_{1}, \ldots, l_{6}, l_{1}^{\prime}, \ldots, l_{6}^{\prime}, l_{12}^{\prime \prime}, l_{13}^{\prime \prime}, \ldots, l_{56}^{\prime \prime}$.

Proposition 1.4. Let $K$ be a field. Then there exists a fine moduli scheme $\widetilde{\mathscr{M}}$ of marked cubic surfaces over $K$, i.e., the functor

$$
\begin{aligned}
F:\{K \text {-schemes }\} & \longrightarrow\{\text { sets }\}, \\
S & \mapsto\{\text { marked cubic surfaces over } S\} / \sim
\end{aligned}
$$

is representable by a $K$-scheme $\widetilde{\mathscr{M}}$.

Sketch of proof. Let $\mathscr{U} \subset\left(\mathbf{P}^{2}\right)^{6}$ be the open subscheme parametrizing all ordered 6 -tuples of points on $\mathbf{P}^{2}$ that are in general position, i.e., no three lie on a line and not all six lie on a conic. $\mathscr{U}$ is acted upon, in an obvious manner, by the algebraic group $\mathrm{PGL}_{3}$.

The Hilbert-Mumford numerical criterion [29, Theorem 2.1] immediately implies that every point $p \in \mathscr{U}$ is $\mathrm{PGL}_{3}$-stable. In fact, the nonstable points on $\left(\mathbf{P}^{2}\right)^{6}$ are those corresponding to configurations such that there are at least four points on a line [29, Definition 3.7/Proposition 3.4]. Hence, the quotient scheme $\mathscr{U} / \mathrm{PGL}_{3}$ exists.

It is well known that $\mathscr{U} / \mathrm{PGL}_{3}$ is the desired fine moduli scheme. A formal proof follows the lines of the proof of [2, Theorem IV.13], with the base field replaced by an arbitrary base scheme.

Remarks 1.5. i) $\widetilde{\mathscr{M}}$ is a quasi-projective fourfold. In fact, such quotients are quasiprojective in much more generality [29, Theorem 1.10.ii].

ii) By functoriality, $\widetilde{\mathscr{M}}$ is acted upon by $W\left(E_{6}\right)$. More precisely, every $g \in G$ defines a permutation of the 27 labels. For every base scheme $S$, this defines a map 
$T_{g}(S): F(S) \rightarrow F(S)$, which is natural in $S$. By Yoneda's lemma, that is equivalent to giving a morphism $T_{g}: \widetilde{\mathscr{M}} \rightarrow \widetilde{\mathscr{M}}$. Clearly, $T_{g g^{\prime}}=T_{g} T_{g^{\prime}}$ for $g, g^{\prime} \in W\left(E_{6}\right)$ and $T_{e}=$ id for $e \in W\left(E_{6}\right)$ the neutral element.

The operation of $W\left(E_{6}\right)$ is not free, as cubic surfaces may have automorphisms. It is, however, free on a nonempty Zariski open subset of $\widetilde{\mathscr{M}}$.

Remarks 1.6 (A naive embedding). $\quad$ i) To give a $\bar{K}$-rational point $p$ on the variety $\mathscr{U}$ is equivalent to giving a sequence of six points $p_{1}, \ldots, p_{6} \in \mathbf{P}^{2}(\bar{K})$ in general position. A standard result from projective geometry states that there is a unique $\gamma \in \mathrm{PGL}_{3}(\bar{K})$ mapping $\left(p_{1}, p_{2}, p_{3}, p_{4}\right)$ to the standard basis $((1: 0: 0)$, $(0: 1: 0),(0: 0: 1),(1: 1: 1))$. The $\bar{K}$-rational points on $\widetilde{\mathscr{M}}$ may thus be represented by $3 \times 6$-matrices of the form

$$
\left(\begin{array}{llllll}
1 & 0 & 0 & 1 & w & y \\
0 & 1 & 0 & 1 & x & z \\
0 & 0 & 1 & 1 & 1 & 1
\end{array}\right) .
$$

Observe that the vanishing of the third coordinate of $p_{5}$ would mean that $p_{1}, p_{2}$, and $p_{5}$ were collinear, and similarly for $p_{6}$. Hence, we actually have an open embedding $\widetilde{\mathscr{M}} \hookrightarrow \mathbf{A}^{4}$.

ii) In particular, one sees that $\widetilde{\mathscr{M}}$ is a smooth, affine scheme. Indeed, the image of the naive embedding of $\widetilde{\mathscr{M}}$ in $\mathbf{A}^{4}$ is the complement of a divisor.

Remark 1.7 (Cayley's compactification). The moduli scheme $\widetilde{\mathscr{M}}$ of marked cubic surfaces has its origins in the middle of the 19th century. In principle, it appears in the article [3] of Arthur Cayley. Cayley's approach was as follows.

Every smooth cubic surface over an algebraically closed field has 45 tritangent planes meeting the surface in three lines. Through each line there are five tritangent planes. This leads to a total of 135 cross ratios, which are invariants of the cubic surface, as soon as a marking is fixed on the lines.

It turns out that only 45 of these cross ratios are essentially different, due to constraints within the cubic surfaces. Furthermore, they provide an embedding $\widetilde{\mathscr{M}} \hookrightarrow\left(\mathbf{P}^{1}\right)^{45}$. The image is Cayley's "cross ratio variety". For a more recent treatment of this compactification, we refer the reader to I. Naruki 30].

\section{Coble's COMpactification. The gamma VARIEty}

Coble's irrational invariants.

2.1. An advantage of the algebraic group $\mathrm{SL}_{3}$ over the group $\mathrm{PGL}_{3}$ is that its operation on $\mathbf{P}^{2}$ is linear. This means that $\mathrm{SL}_{3}$ operates naturally on $\mathscr{O}(n)$, and hence on $\Gamma\left(\mathbf{P}^{2}, \mathscr{O}(n)\right)$, for every $n$. It is well known that there is no $\mathrm{PGL}_{3}$-linearization for $\mathscr{O}_{\mathbf{P}^{2}}(1)$ [29, Chapter $\left.1, \S 3\right]$.

There is, however, the canonical isogeny $\mathrm{SL}_{3} \rightarrow \mathrm{PGL}_{3}$, the kernel of which consists of the multiples of the identity matrix by the third roots of unity. These matrices clearly operate trivially on $\mathscr{O}(3)$. Thus, there is a canonical $\mathrm{PGL}_{3}$-linearization for $\mathscr{O}(3)$, which is compatible with the $\mathrm{SL}_{3}$-linearization; cf. [29, Chapter $3, \S 1$ ].

We may also speak of $\mathrm{SL}_{3}$-invariant sections of the outer tensor products $\mathscr{O}\left(n_{1}\right) \otimes \ldots \otimes \mathscr{O}\left(n_{6}\right)$ on $\left(\mathbf{P}^{2}\right)^{6}$ for $\left(n_{1}, \ldots, n_{6}\right) \in \mathbb{Z}^{6}$. If $3 \mid n_{1}, \ldots, n_{6}$, then $\mathrm{PGL}_{3}$ operates, too, and the $\mathrm{PGL}_{3}$-invariant sections are the same as the $\mathrm{SL}_{3}$-invariant ones. 
2.2. For example, for $1 \leq i_{1}<i_{2}<i_{3} \leq 6$, the corresponding minor

$$
m_{\left\{i_{1}, i_{2}, i_{3}\right\}}:=\operatorname{det}\left(\begin{array}{lll}
x_{i_{1}, 0} & x_{i_{1}, 1} & x_{i_{1}, 2} \\
x_{i_{2}, 0} & x_{i_{2}, 1} & x_{i_{2}, 2} \\
x_{i_{3}, 0} & x_{i_{3}, 1} & x_{i_{3}, 2}
\end{array}\right)
$$

of the $6 \times 3$-matrix

$$
\left(\begin{array}{ccc}
x_{1,0} & x_{1,1} & x_{1,2} \\
x_{2,0} & x_{2,1} & x_{2,2} \\
& \cdots & \\
x_{6,0} & x_{6,1} & x_{6,2}
\end{array}\right)
$$

defines an invariant section of $\mathscr{O}\left(n_{1}\right) \otimes \ldots \otimes \mathscr{O}\left(n_{6}\right)$ for

$$
n_{i}:=\left\{\begin{array}{l}
1 \text { for } i \in\left\{i_{1}, i_{2}, i_{3}\right\} \\
0 \text { for } i \notin\left\{i_{1}, i_{2}, i_{3}\right\}
\end{array} .\right.
$$

Further,

$$
d_{2}:=\operatorname{det}\left(\begin{array}{cccccc}
x_{1,0}^{2} & x_{1,1}^{2} & x_{1,2}^{2} & x_{1,0} x_{1,1} & x_{1,0} x_{1,2} & x_{1,1} x_{1,2} \\
x_{2,0}^{2} & x_{2,1}^{2} & x_{2,2}^{2} & x_{2,0} x_{2,1} & x_{2,0} x_{2,2} & x_{2,1} x_{2,2} \\
5 & \ldots & & \\
x_{6,0}^{2} & x_{6,1}^{2} & x_{6,2}^{2} & x_{6,0} x_{6,1} & x_{6,0} x_{6,2} & x_{6,1} x_{6,2}
\end{array}\right) \in \Gamma\left(\left(\mathbf{P}^{2}\right)^{6}, \mathscr{O}(2) \otimes \ldots \otimes \mathscr{O}(2)\right)
$$

is $\mathrm{SL}_{3}$-invariant, too.

A. Coble [6, formulas (16) and (18)] now defines $40 \mathrm{SL}_{3}$-invariant, and hence $\mathrm{PGL}_{3}$-invariant, sections $\gamma . \in \Gamma\left(\left(\mathbf{P}^{2}\right)^{6}, \mathscr{O}(3) \otimes \ldots \otimes \mathscr{O}(3)\right)$.

Definition 2.3 (Coble). For $\left\{i_{1}, \ldots, i_{6}\right\}=\{1, \ldots, 6\}$, consider

$$
\begin{aligned}
\gamma_{\left(i_{1} i_{2} i_{3}\right)\left(i_{4} i_{5} i_{6}\right)} & :=m_{\left\{i_{1}, i_{2}, i_{3}\right\}} m_{\left\{i_{4}, i_{5}, i_{6}\right\}} d_{2} \quad \text { and } \\
\gamma_{\left(i_{1} i_{2}\right)\left(i_{3} i_{4}\right)\left(i_{5} i_{6}\right)} & :=m_{\left\{i_{1}, i_{3}, i_{4}\right\}} m_{\left\{i_{2}, i_{3}, i_{4}\right\}} m_{\left\{i_{3}, i_{5}, i_{6}\right\}} m_{\left\{i_{4}, i_{5}, i_{6}\right\}} m_{\left\{i_{5}, i_{1}, i_{2}\right\}} m_{\left\{i_{6}, i_{1}, i_{2}\right\}} .
\end{aligned}
$$

Following the original work, we will call these 40 sections the irrational invariants.

Remarks 2.4. i) Here, the combinatorial structure is as follows. Within the parentheses, the indices may be arbitrarily permuted without changing the symbol. Further, in the symbols $\gamma_{\left(i_{1} i_{2} i_{3}\right)\left(i_{4} i_{5} i_{6}\right)}$, the two triples may be interchanged. However, in the symbols $\gamma_{\left(i_{1} i_{2}\right)\left(i_{3} i_{4}\right)\left(i_{5} i_{6}\right)}$, the three pairs may be permuted only cyclically. Thus, altogether, there are ten invariants of the first type and 30 invariants of the second type.

ii) The 20 minors $m_{\left\{i_{1}, i_{2}, i_{3}\right\}}$ and the invariant $d_{2}$ vanish only when the underlying six points $\left(x_{1}, \ldots, x_{6}\right)$ are not in general position. Hence, on $\mathscr{U}$, Coble's 40 sections have no zeroes.

iii) One has the beautiful relation

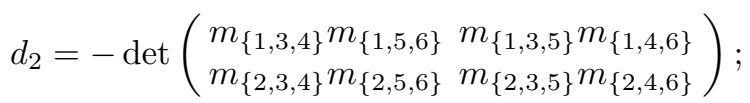

cf. [5, (47)] or [21, formula (4.18)].

Caution 2.5. We have 40 sections $\gamma . \in \Gamma\left(\left(\mathbf{P}^{2}\right)^{6}, \mathscr{O}(3) \otimes \ldots \otimes \mathscr{O}(3)\right)^{\mathrm{PGL}_{3}}$, and a machine calculation shows $\operatorname{dim} \Gamma\left(\left(\mathbf{P}^{2}\right)^{6}, \mathscr{O}(3) \otimes \ldots \otimes \mathscr{O}(3)\right)^{\mathrm{PGL}_{3}}=40$.

It is, however, long known [6, (24)] that the 40 sections $\gamma$. span only a subvector space of dimension ten. The mere fact that there is such a gap is quite obvious. In fact, for $\left(p_{1}, \ldots, p_{6}\right) \in\left(\mathbf{P}^{2}\right)^{6}$ such that $p_{1}, \ldots, p_{4}$ are distinct points on a line $l$ and $p_{5}, p_{6} \notin l$, we have $m_{\{1,2,5\}}^{3} m_{\{3,4,6\}}^{3} \neq 0$ but all $\gamma$. vanish. 
In particular, the irrational invariants $\gamma$. do not generate the invariant ring

$$
\bigoplus_{d \geq 0} \Gamma\left(\left(\mathbf{P}^{2}\right)^{6}, \mathscr{O}(3 d) \otimes \ldots \otimes \mathscr{O}(3 d)\right)^{\mathrm{PGL}_{3}}
$$

and do not define an embedding of the categorical quotient $\left(\left(\mathbf{P}^{2}\right)^{6}\right)^{\mathrm{ss}} / \mathrm{PGL}_{3}$ [29, Definition 0.5] into $\mathbf{P}^{39}$. Observe, however, Theorem 2.7 below.

Notation 2.6. The $\mathrm{PGL}_{3}$-invariant local sections of $\mathscr{O}(3) \otimes \ldots \otimes \mathscr{O}(3)$ form an invertible sheaf on $\widetilde{\mathscr{M}}=\mathscr{U} / \mathrm{PGL}_{3}$, which we will denote by $\mathscr{L}$.

Theorem 2.7. a) The invertible sheaf $\mathscr{L}$ on $\widetilde{\mathscr{M}}$ is very ample.

b) The 40 irrational invariants $\gamma . \in \Gamma(\widetilde{\mathscr{M}}, \mathscr{L})$ define a projective embedding $\gamma: \widetilde{\mathscr{M}} \hookrightarrow \mathbf{P}_{K}^{39}$.

c) The Zariski closure $\widetilde{M}$ of the image of $\gamma$ is contained in a nine-dimensional linear subspace.

d) As a subvariety of this $\mathbf{P}^{9}, \widetilde{M}$ has the properties below.

i) The image of $\widetilde{M}$ under the 2-uple Veronese embedding $\mathbf{P}^{9} \hookrightarrow \mathbf{P}^{54}$ is not contained in any proper linear subspace.

ii) The image of $\widetilde{M}$ under the 3-uple Veronese embedding $\mathbf{P}^{9} \hookrightarrow \mathbf{P}^{219}$ is contained in a linear subspace of dimension 189.

iii) $\widetilde{M}$ is the intersection of 30 cubic hypersurfaces.

Proof. Assertion b) is [7, Corollary 5.9]. The proof given there is based on the considerations of I. Naruki 30. a) is clearly implied by b).

c) follows from the fact that the vector space $\langle\gamma$. $\rangle$ spanned by the 40 irrational invariants $\gamma$. is ten-dimensional.

d.i) and ii) As is easily checked by computer, the purely quadratic expressions in the $\gamma$. form a 55-dimensional vector space, while the purely cubic expressions form a vector space of dimension 190 .

iii) By ii), $\widetilde{M}$ is contained in the intersection of 30 cubic hypersurfaces in $\mathbf{P}^{9}$. This intersection is reported by magma as being reduced and irreducible of dimension four.

Remarks 2.8. i) As $\gamma: \widetilde{\mathscr{M}} \rightarrow \mathbf{P}_{K}^{39}$ is a map that is given completely explicitly, one might try to use computer algebra to prove it is an embedding. This indeed works, at least when one organizes the computation in a slightly deliberate way.

It turns out that the composition $\widetilde{\gamma}$ of $\gamma$ with the linear projection to the $\mathbf{P}^{9}$, formed by the ten invariants of type $\gamma_{\left(i_{1} i_{2} i_{3}\right)\left(i_{4} i_{5} i_{6}\right)}$, is already defined everywhere and separates tangent vectors. It is a $2: 1$-morphism identifying $(w, x, y, z)$ with $\left(w^{\prime}, x^{\prime}, y^{\prime}, z^{\prime}\right)$ for

$$
\begin{aligned}
& w^{\prime}:=\frac{(w z-x y)(z-1)}{(x-z)(y-z)}, \\
& x^{\prime}:=\frac{(w z-x y)(y-1)}{(w-y)(y-z)}, \\
& y^{\prime}:=\frac{(w z-x y)(x-1)}{(w-x)(x-z)}, \\
& z^{\prime}:=\frac{(w z-x y)(w-1)}{(w-x)(w-y)} .
\end{aligned}
$$

A Gröbner base calculation in four variables readily shows that these two points never have the same image under $\gamma$.

ii) The partner point $\left(w^{\prime}, x^{\prime}, y^{\prime}, z^{\prime}\right)$ corresponds to the same cubic surface as $(w, x, y, z)$, but with the flipped marking, i.e., $l_{i}$ is replaced by $l_{i}^{\prime}$ and vice versa. This is seen by a short calculation from [6, Table (2)]; cf. [5, p. 196]. 
Definitions 2.9. i) We will call $\gamma: \widetilde{\mathscr{M}} \hookrightarrow \mathbf{P}_{K}^{39}$ Coble's gamma map.

ii) The variety $\widetilde{M}$, given as the Zariski closure of the image of $\gamma$, will be called Coble's gamma variety.

Remarks 2.10. i) The fact that the vector space $\langle\gamma$. $\rangle$ is only of dimension ten is, of course, easily checked by computer, as well.

ii) The assertions c) and d) are due to A. Coble himself. For d), we advise the reader to compare the result [1, Theorem 6.4] of D. Allcock and E. Freitag.

Coble's original proof for c) works as follows [6, (24)]. One may write down [6, page 343] five four-term linear relations, the $S_{6}$-orbits of which yield a total of 270 relations. These relations form a single orbit under $W\left(E_{6}\right)$ and generate the 30-dimensional space of all linear relations.

In order to show that the dimension is not lower than ten, Coble has to use the moduli interpretation. He verifies that there are enough cubic surfaces in hexahedral form.

iii) The cubic relations are in fact more elementary than the linear ones. For example, one has

$$
\gamma_{(12)(34)(56)} \gamma_{(23)(45)(16)} \gamma_{(14)(36)(25)}=\gamma_{(12)(36)(45)} \gamma_{(34)(25)(16)} \gamma_{(56)(14)(23)} .
$$

To see this, look at the left hand side first. The nine pairs of numbers in $\{1, \ldots, 6\}$ that are used, are exactly those with an odd difference. Thus, when writing, according to the very definition, the left side as a product of 18 minors, $m_{1,3,5}$ and $m_{2,4,6}$ cannot appear. It turns out that each of the other minors occurs exactly once. As the same is true for the right hand side, the equality becomes evident.

We remark that this relation is not a consequence of the linear ones, i.e., it does not become trivial when restricted to $\mathbf{P}^{9}$. Its orbit under $W\left(E_{6}\right)$ must generate the 30-dimensional space of all cubic relations. Indeed, that is an irreducible representation, as we will show in the next subsection.

iv) In particular, the gamma variety $\widetilde{M}$ is clearly not a complete intersection. Nevertheless, the following of its numerical invariants may be computed.

Lemma 2.11. i) The Hilbert series of $\widetilde{M}$ is $\frac{1+5 T+15 T^{2}+5 T^{3}+T^{4}}{(1-T)^{5}}$.

ii) In particular, the Hilbert polynomial of $\widetilde{M}$ is $\frac{9}{8} T^{4}+\frac{9}{4} T^{3}+\frac{27}{8} T^{2}+\frac{9}{4} T+1$. Further, the Hilbert polynomial agrees with the Hilbert function in all degrees $\geq 0$.

iii) $\widetilde{M}$ is a projective variety of degree 27 .

iv) The Castelnuovo-Mumford regularity of $\widetilde{M}$ is equal to 4 and that of the ideal sheaf $\mathscr{I}_{\widetilde{M}} \subset \mathscr{O}_{\mathbf{P}^{39}}$ is equal to 5 .

Proof. i) follows from a Gröbner base calculation. ii) and iii) are immediate consequences of i).

iv) By [8, p. 219], it is pure linear algebra to compute the Castelnuovo-Mumford regularity of a coherent $\mathscr{O}_{\mathbf{P}^{N}}$-module. We used the implementation in magma.

The operation of $W\left(E_{6}\right)$.

2.12. It is an important feature of Coble's (as well as Cayley's) compactifications that they explicitly linearize the operation of $W\left(E_{6}\right)$. More precisely,

Lemma. There exists a $W\left(E_{6}\right)$-linearization of $\mathscr{L} \in \operatorname{Pic}(\widetilde{\mathscr{M}})$ such that

i) The 80 sections $\pm \gamma$. $\in \Gamma(\widetilde{\mathscr{M}}, \mathscr{L})$ form a $W\left(E_{6}\right)$-invariant set. 
ii) The corresponding permutation representation $\Pi: W\left(E_{6}\right) \hookrightarrow S_{80}$ is transitive. It has a system of 40 blocks given by the pairs $\{\gamma,-\gamma\}$.

iii) The permutation representation $W\left(E_{6}\right) \hookrightarrow S_{40}$ on the 40 blocks is the same as that on decompositions of the 27 lines into three pairs of Steiner trihedra.

Proof. i) (Cf. 7. Section 2].) As $W\left(E_{6}\right)$ is a discrete group, the general concept of a linearization of an invertible sheaf 29, Definition 1.6] breaks down to a system of compatible isomorphisms $i_{g}: T_{g}^{*} \mathscr{L} \stackrel{\stackrel{\varrho}{\longrightarrow}}{\longrightarrow} \mathscr{L}$ for $T_{g}: \widetilde{\mathscr{M}} \rightarrow \widetilde{\mathscr{M}}$ the operation of $g$.

For $g \in S_{6} \subset W\left(E_{6}\right)$, there is an obvious such isomorphism. Indeed, $g$ permutes the six labels $l_{1}, \ldots, l_{6}$ and, accordingly, the six blow-up points $p_{1}, \ldots, p_{6}$. Simply permute the six factors of $\mathscr{O}(3) \otimes \ldots \otimes \mathscr{O}(3)$ as described by $g$. Assertion i) is clear for these elements.

Further, $W\left(E_{6}\right)$ is generated by $S_{6}$ and just one additional element, the quadratic transformation $I_{123}$ with centre in $p_{1}, p_{2}$, and $p_{3}$ [20. Example V.4.2.3]. In the coordinates described in Remark 1.6 i), this map is given by $(w, x, y, z) \mapsto\left(\frac{1}{w}, \frac{1}{x}, \frac{1}{y}, \frac{1}{z}\right)$.

One may now list explicit formulas for the 40 irrational invariants $\gamma$. in terms of these coordinates. Each of these sections actually defines a global trivialization of $\mathscr{L}$. Plugging in the provision $(w, x, y, z) \mapsto\left(\frac{1}{w}, \frac{1}{x}, \frac{1}{y}, \frac{1}{z}\right)$ in a naive way yields an isomorphism $i_{I_{123}}^{\prime}: T_{I_{123}}^{*} \mathscr{L} \stackrel{\mathscr{L}}{\cong}$. It turns out that, under $i_{I_{123}}^{\prime}$, the 40 sections $\gamma$. are permuted up to signs and a common scaling factor of $\frac{1}{w^{2} x^{2} y^{2} z^{2}}$. Thus, let us take $i_{I_{123}}:=w^{2} x^{2} y^{2} z^{2} \cdot i_{I_{123}}^{\prime}$ as the actual definition.

This uniquely determines $i_{g}$ for every $g \in W\left(E_{6}\right)$. One may check that $\left\{i_{g}\right\}_{g \in W\left(E_{6}\right)}$ is a well-defined linearization of $\mathscr{L}$. Assertion i) is then clear.

ii) We checked the first assertion in magma. The second statement is obvious.

iii) Note that, in the blown-up model, the 40 irrational invariants have exactly the same combinatorial structure as the 40 decompositions; cf. [16, 3.7].

Remarks 2.13. i) The permutation representation $\Pi$ has no other nontrivial block structures.

ii) The restriction of $\Pi$ to the index-two subgroup $D^{1} W\left(E_{6}\right) \subset W\left(E_{6}\right)$, which is the simple group of order 25920 , is still transitive. Neither does it have more block structures.

iii) Lemma 2.12, i) suggests that it might have technical advantages to consider the embedding $\gamma^{\prime}: \mathscr{M} \hookrightarrow \mathbf{P}^{79}$, linearly equivalent to the gamma map $\gamma$, which is defined by the 80 sections $\pm \gamma$. To a certain extent, this is indeed the case; cf. Remarks 4.4

Remarks 2.14 (Representations of $W\left(E_{6}\right)$ ). i) The dimensions of the irreducible complex representations of $W\left(E_{6}\right)$ are $1,1,6,6,10,15,15,15,15,20,20,20,24$, $24,30,30,60,60,60,64,64,80,81,81$, and 90 .

ii) The $W\left(E_{6}\right)$-representation on the vector space $V:=\langle\gamma.\rangle \cong \Gamma\left(\mathbf{P}^{9}, \mathscr{O}(1)\right)$ of dimension ten is irreducible.

iii) The $W\left(E_{6}\right)$-representation on the 220 -dimensional vector space $\Gamma\left(\mathbf{P}^{9}, \mathscr{O}(3)\right)$ decomposes into two copies of the ten-dimensional, two copies of a 30-dimensional, two copies of the other 30-dimensional, and one copy of the 80-dimensional irreducible representations [9, Theorem 3.2.2]. This already implies that the 30dimensional subrepresentation of cubic relations among the $\gamma$. is irreducible.

Remarks 2.15. a) The embedding of the moduli scheme of marked cubic surfaces into $\mathbf{P}^{9}$, originally due to A. B. Coble, was studied recently by D. Allcock and E. Freitag [1, as well as B. van Geemen [18. Their approaches were rather different 
from Coble's. For example, van Geemen actually constructs an embedding of the cross ratio variety, instead of $\mathscr{U} / \mathrm{PGL}_{3}$, into $\mathbf{P}^{9}$. He obtains the 30 cubic relations in [18, 7.9].

b) A short summary of Coble's approach may be found in I. Dolgachev's book on classical algebraic geometry [10, Remark 9.4.20].

\section{The MODUli SCHEME OF UN-MARKED CUBIC SURFACES}

3.1. The quotient $\widetilde{\mathscr{M}} / W\left(E_{6}\right)=: \mathscr{M}$ is the coarse moduli scheme of smooth cubic surfaces. The reader might consult [30, Appendix by E. Looijenga] for more details on this quotient. As cubic surfaces may have automorphisms, a fine moduli scheme cannot exist.

3.2. The moduli scheme of smooth cubic surfaces may as well be constructed directly as the quotient $\mathscr{V} / \mathrm{PGL}_{4}$, for $\mathscr{V} \subset \mathbf{P}\left(\operatorname{Sym}^{3}\left(K^{4}\right)^{*}\right) \cong \mathbf{P}^{19}$ the open subscheme parametrizing smooth cubic surfaces. In fact, by [28, 1.14], every smooth cubic surface corresponds to a $\mathrm{PGL}_{4}$-stable point on $\mathbf{P}^{19}$.

The $\mathrm{PGL}_{4}$-invariants were determined by A. Clebsch [4, sections 4 and 5] as early as 1861. In today's language, Clebsch's result is that there is an open embedding $\mathrm{Cl}: \mathscr{V} / \mathrm{PGL}_{4} \cong \mathscr{M} \hookrightarrow \mathbf{P}(1,2,3,4,5)$ into a weighted projective space [10, formula (9.57)].

Definition 3.3. i) The homogeneous coordinates on $\mathbf{P}(1,2,3,4,5)$ will be denoted, in this order, by $A, B, C, D$, and $E$.

ii) Thus, given a smooth cubic surface over a field $K$, there is the corresponding $K$-rational point on $\mathbf{P}(1,2,3,4,5)$. Its homogeneous coordinates form a vector $[A, \ldots, E]$, which is unique up to weighted scaling, for the weight vector $(1, \ldots, 5)$. We will speak of Clebsch's invariant vector or simply Clebsch's invariants of the cubic surface.

Example 3.4. Consider the pentahedral family $\mathscr{C} \rightarrow \mathbf{P}^{4} / S_{5}$ of cubic surfaces, given by

$$
\begin{aligned}
a_{0} X_{0}^{3}+a_{1} X_{1}^{3}+a_{2} X_{2}^{3}+a_{3} X_{3}^{3}+a_{4} X_{4}^{3}=0 \\
X_{0}+X_{1}+X_{2}+X_{3}+X_{4}=0
\end{aligned}
$$

over $\mathbf{P}^{4} / S_{5} \cong \mathbf{P}(1,2,3,4,5)$. We will use the elementary symmetric functions $\sigma_{1}, \ldots, \sigma_{5}$ in $a_{0}, \ldots, a_{4}$ as natural homogeneous coordinates on $\mathbf{P}^{4} / S_{5}$.

Let us restrict our considerations to the open subset $\mathscr{P} \subset \mathbf{P}^{4} / S_{5}$ representing smooth cubic surfaces having a proper pentahedron. The latter condition is equivalent to $\sigma_{5} \neq 0$.

Then, for $t: \mathscr{P} \longrightarrow \mathscr{M}$ the classifying morphism, the composition Clot: $\mathscr{P} \rightarrow \mathscr{M} \hookrightarrow \mathbf{P}(1,2,3,4,5)$ is given by the $S_{5}$-invariant sections

$$
I_{8}:=\sigma_{4}^{2}-4 \sigma_{3} \sigma_{5}, \quad I_{16}:=\sigma_{1} \sigma_{5}^{3}, \quad I_{24}:=\sigma_{4} \sigma_{5}^{4}, \quad I_{32}:=\sigma_{2} \sigma_{5}^{6}, \quad I_{40}:=\sigma_{5}^{8}
$$

of $\mathscr{O}(8), \mathscr{O}(16), \mathscr{O}(24), \mathscr{O}(32)$, and $\mathscr{O}(40)$, respectively. See [10, formula (9.59)] or [31, paragraph 543]. In other words $(\mathrm{Cl} \circ t)^{-1}(A)=I_{8}, \ldots,(\mathrm{Cl} \circ t)^{-1}(E)=I_{40}$.

Lemma 3.5. The classifying morphism $t: \mathscr{P} \rightarrow \mathscr{M}$ is an open embedding. 
Proof. It will suffice to show that Clot: $\mathscr{P} \rightarrow \mathbf{P}(1,2,3,4,5)$ is an open embedding. For this, we first observe that Clot is birational. Indeed, the two function fields are

$$
\begin{aligned}
K(\mathbf{P}(1,2,3,4,5)) & =K\left(B / A^{2}, C / A^{3}, D / A^{4}, E / A^{5}\right) \\
& =K\left(A^{2} / B, A^{3} / C, A^{4} / D, A^{5} / E\right)
\end{aligned}
$$

and $K(\mathscr{P})=K\left(\sigma_{2} / \sigma_{1}^{2}, \sigma_{3} / \sigma_{1}^{3}, \sigma_{4} / \sigma_{1}^{4}, \sigma_{5} / \sigma_{1}^{5}\right)$. Both are of transcendence degree four over $K$.

Consider the finitely generated $K$-algebra

$$
R:=K\left[\frac{A^{2}}{B}, \frac{A^{3}}{C}, \frac{A^{4}}{D}, \frac{A^{5}}{E}, \frac{D}{B^{2}}, \frac{C^{2}-A E}{4 B^{3}}, \frac{C E}{B^{4}}, \frac{E^{2}}{B^{5}}\right],
$$

which is a subdomain of $K(\mathbf{P}(1,2,3,4,5))$. The formulas (3.2) together with

$$
\begin{aligned}
(\mathrm{Cl} \circ t)^{-1}\left(\frac{D}{B^{2}}\right) & =\sigma_{2} / \sigma_{1}^{2}, & (\mathrm{Cl} \circ t)^{-1}\left(\frac{C^{2}-A E}{4 B^{3}}\right) & =\sigma_{3} / \sigma_{1}^{3}, \\
(\mathrm{Cl} \circ t)^{-1}\left(\frac{C E}{B^{4}}\right) & =\sigma_{4} / \sigma_{1}^{4}, & (\mathrm{Cl} \circ t)^{-1}\left(\frac{E^{2}}{B^{5}}\right) & =\sigma_{5} / \sigma_{1}^{5},
\end{aligned}
$$

immediately define a $K$-algebra homomorphism $\iota: R \rightarrow K(\mathscr{P})$. For $\mathfrak{p}:=\operatorname{ker} \iota$, we have a homomorphism $\mathrm{Q}(R / \mathfrak{p}) \hookrightarrow K(\mathscr{P})$ of fields.

As $\sigma_{2} / \sigma_{1}^{2}, \sigma_{3} / \sigma_{1}^{3}, \sigma_{4} / \sigma_{1}^{4}$, and $\sigma_{5} / \sigma_{1}^{5}$ are in the image, we see that $(\mathrm{Cl} \circ t)^{-1}$ actually defines an isomorphism $\mathrm{Q}(R / \mathfrak{p}) \cong K(\mathscr{P})$. In particular, $\mathrm{Q}(R / \mathfrak{p})$ is of transcendence degree four and, consequently, $\mathfrak{p}=(0)$. As $\mathrm{Q}(R)=K(\mathbf{P}(1,2,3,4,5))$, the claim follows.

Furthermore, Clot is a quasi-finite morphism. In fact, this may be tested on closed points and after base extension to the algebraic closure $\bar{K}$. Thus, let $p=$ $(A, \ldots, E) \in \mathbf{P}(1,2,3,4,5)(\bar{K})$ be a geometric point. If $E=0$, then $(\mathrm{Cl} \circ t)^{-1}(p)=$ $\emptyset$. Otherwise, there are eight solutions of $\sigma_{5}^{8}=E$ and, for each choice, $\sigma_{1}, \ldots, \sigma_{4}$ may be computed directly.

Finally, $\mathbf{P}(1,2,3,4,5)$ is a toric variety [17, section 2.2, page 35] and hence a normal scheme [17, section 2.1, page 29]. Therefore the assertion is implied by [19, Corollaire (4.4.9)].

Remarks 3.6. i) In particular, a general cubic surface over a field has a proper pentahedron, which will usually be defined over a finite extension field.

ii) Further, on the open subset of $\mathscr{M}$ representing smooth cubic surfaces with a proper pentahedron, $\sigma_{1}, \ldots, \sigma_{5}$ serve well as coordinates. It is highly remarkable that they do not extend properly to the whole of $\mathscr{M}$.

Example 3.7. There are other prominent families of smooth cubic surfaces. The most interesting ones are probably the hexahedral families. Consider $\mathscr{C} \rightarrow H \subset \mathbf{P}^{5}$, where $\mathscr{C} \subset H \times \mathbf{P}^{4}$ is given by

$$
\begin{gathered}
X_{0}^{3}+X_{1}^{3}+X_{2}^{3}+X_{3}^{3}+X_{4}^{3}+X_{5}^{3}=0 \\
X_{0}+X_{1}+X_{2}+X_{3}+X_{4}+X_{5}=0 \\
a_{0} X_{0}+a_{1} X_{1}+a_{2} X_{2}+a_{3} X_{3}+a_{4} X_{4}+a_{5} X_{5}=0
\end{gathered}
$$

and $H \subset \mathbf{P}^{5}$ is the hyperplane defined by $a_{0}+\ldots+a_{5}=0$. This is the ordered hexahedral family of cubic surfaces. Correspondingly, the base of the unordered hexahedral family is the quotient $H / S_{6} \cong \mathbf{P}(2,3,4,5,6)$.

There are the tautological morphisms $\stackrel{\mathscr{M}}{\longrightarrow} H \stackrel{t_{1}}{\longrightarrow} H / S_{6} \stackrel{t_{3}}{\longrightarrow} \mathscr{M}$. It is classically known that $t_{1}$ is an unramified $2: 1$-covering and that $t_{3}$ is an unramified $36: 1$-covering. Clearly, $t_{2}$ is generically $720: 1$. 
Example 3.8 (continued). It seems natural to use the elementary symmetric functions $\sigma_{2}, \ldots, \sigma_{6}$ in the hexahedral coefficients as homogeneous coordinates on $H / S_{6}$. Then it is possible, today, to give explicit formulas for the composition $\mathrm{Clot}_{3}: H / S_{6} \rightarrow \mathscr{M} \hookrightarrow \mathbf{P}(1,2,3,4,5)$.

This means to convert the formulas (3.2) for Clebsch's invariants to the hexahedral form. The first of these formulas,

$$
\left(\mathrm{Cl} \circ t_{3}\right)^{-1}(A)=24\left[4 \sigma_{2}^{3}-3 \sigma_{3}^{2}-16 \sigma_{2} \sigma_{4}+12 \sigma_{6}\right],
$$

was established by C. P. Sousley [34, formula (17)], back in 1917. Here, the coefficient 24 is somewhat conventional, as it depends on the choice of an isomorphism $\left(\mathrm{Cl}_{3}\right) * \mathscr{O}(1) \cong \mathscr{O}(6)$.

Formula (3.3) agrees with the modern treatment, due to I. V. Dolgachev 10, Remark 9.4.19] as well as with [21, formula (B.56)]. Other coefficients were used, however, in Coble's original work [6, formula (9)] and to obtain [21, formula (4.108)].

Theorem 3.9. i) The canonical morphism

$$
\psi: \widetilde{\mathscr{M}} \stackrel{\mathrm{pr}}{\longrightarrow} \mathscr{M} \stackrel{\mathrm{Cl}}{\hookrightarrow} \mathbf{P}(1,2,3,4,5)
$$

allows an extension to $\mathbf{P}^{39}$ under the gamma map. More precisely, there exists a rational map $\widetilde{\psi}: \mathbf{P}^{39}{ }_{-} \rightarrow \mathbf{P}(1,2,3,4,5)$ such that the following diagram commutes:

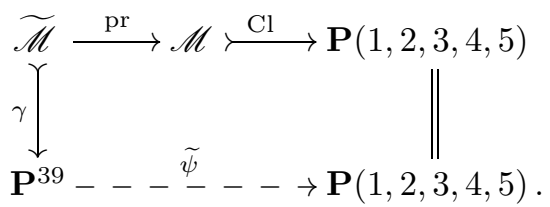

ii) Explicitly, the rational map $\widetilde{\psi}: \mathbf{P}^{39}{ }_{-} \rightarrow \mathbf{P}(1,2,3,4,5)$, defined by the global sections

$$
\begin{aligned}
& \text { - }-6 P_{2} \in \Gamma\left(\mathbf{P}^{39}, \mathscr{O}(2)\right), \\
& \text { - }-24 P_{4}+\frac{41}{16} P_{2}^{2} \in \Gamma\left(\mathbf{P}^{39}, \mathscr{O}(4)\right), \\
& \text { - } \frac{576}{13} P_{6}-\frac{396}{13} P_{4} P_{2}+\frac{29}{13} P_{2}^{3} \in \Gamma\left(\mathbf{P}^{39}, \mathscr{O}(6)\right), \\
& \text { - }-\frac{62208}{1171} P_{8}+\frac{54864}{1171} P_{6} P_{2}+\frac{203616}{1171} P_{4}^{2}-\frac{61287}{1171} P_{4} P_{2}^{2} \\
& \quad+\frac{13393}{4684} P_{2}^{4} \in \Gamma\left(\mathbf{P}^{39}, \mathscr{O}(8)\right), \\
& \text { - } \frac{41472}{155} P_{10}-\frac{4605984}{36301} P_{8} P_{2}-\frac{106272}{403} P_{6} P_{4}+\frac{19990440}{471913} P_{6} P_{2}^{2}+\frac{47719206}{471913} P_{4}^{2} P_{2} \\
& \quad-\frac{7468023}{471913} P_{4} P_{2}^{3}+\frac{10108327}{18876520} P_{2}^{5} \in \Gamma\left(\mathbf{P}^{39}, \mathscr{O}(10)\right),
\end{aligned}
$$

satisfies this condition. Here, $P_{k}$ denotes the sum of the $40 k$-th powers.

iii) In other words, these formulas express Clebsch's invariants $A, \ldots, E$ in terms of Coble's 40 irrational invariants $\gamma$.

Remarks 3.10. i) The formula for the first Clebsch invariant is due to A. B. Coble; cf. [6, formula (38)] and [21, formula (4.108)]. It may be obtained by plugging the formula [5, formula (85)], computing hexahedral coefficients out of six blow-up points, into Sousley's formula (3.3).

ii) Similarly to Example 3.8, there is a minor ambiguity here, due to the possibility of scaling. The coefficient $(-6)$ in the first formula agrees with Sousley's formula (3.3). 
3.11. Proof of Theorem 3.9, We will prove this theorem in several steps.

First step. Results from the literature and preparations.

A considerable part of this result is available from the literature. First of all, it is known that the morphism $\psi:=\mathrm{Cl} \circ \mathrm{pr}$ in the upper row extends to a finite morphism $\varphi: \widetilde{M} \rightarrow \mathbf{P}(1,2,3,4,5)$ from the gamma variety [7, Proposition 1.3].

Further,

$$
\left.\varphi^{*} \mathscr{O}(1) \cong \mathscr{O}(2)\right|_{\widetilde{M}} .
$$

Indeed, this follows from the functoriality of the determinant line bundle, 7, Definition 1.1], together with its calculation for both sides, [7, Proposition 1.3] and 7. Section 2].

The fact that $\varphi: \widetilde{M} \rightarrow \mathbf{P}(1,2,3,4,5)$ is a finite morphism ensures that

$$
\varphi^{-1}: \Gamma(\mathbf{P}(1,2,3,4,5), \mathscr{O}(i)) \longrightarrow \Gamma\left(\widetilde{M},\left.\mathscr{O}(2 i)\right|_{\widetilde{M}}\right)^{W\left(E_{6}\right)}
$$

is a bijection for each $i$. In particular, every $W\left(E_{6}\right)$-invariant even degree homogeneous polynomial expression in Coble's irrational invariants induces an element of Clebsch's invariant ring.

Second step. Extending the sections to $\mathbf{P}^{39}$.

Unfortunately, we need exactly the opposite. To ensure this, we claim that the restriction map

$$
\operatorname{res}_{i}: \Gamma\left(\mathbf{P}^{39}, \mathscr{O}(2 i)\right)^{W\left(E_{6}\right)} \rightarrow \Gamma\left(\widetilde{M},\left.\mathscr{O}(2 i)\right|_{\widetilde{M}}\right)^{W\left(E_{6}\right)}
$$

is surjective, for $i=1, \ldots, 5$.

In view of the bijectivity of $\varphi^{-1}$, it will suffice to verify that $\operatorname{dim} \operatorname{im} \operatorname{res}_{i} \geq d_{i}$ for

$$
d_{i}:= \begin{cases}1 & \text { for } i=1 \\ 2 & \text { for } i=2 \\ 3 & \text { for } i=3 \\ 5 & \text { for } i=4 \\ 7 & \text { for } i=5\end{cases}
$$

For this, let us write some $W\left(E_{6}\right)$-invariant sections of $\left.\mathscr{O}(2 i)\right|_{\widetilde{M}}$ that are contained in the image of $\operatorname{res}_{i}$. Denote by $P_{i}:=\sum_{j=0}^{39} X_{j}^{i}$ the $i$-th power sum. Then

$$
\begin{aligned}
& \operatorname{im}\left(\operatorname{res}_{i}: \Gamma\left(\mathbf{P}^{39}, \mathscr{O}(2 i)\right)^{W\left(E_{6}\right)} \rightarrow \Gamma\left(\widetilde{M},\left.\mathscr{O}(2 i)\right|_{\widetilde{M}}\right)^{W\left(E_{6}\right)}\right) \\
& \supseteq \begin{cases}\left\langle P_{2}\right\rangle & \text { for } i=1, \\
\left\langle P_{4}, P_{2}^{2}\right\rangle & \text { for } i=2, \\
\left\langle P_{6}, P_{4} P_{2}, P_{2}^{3}\right\rangle & \text { for } i=3, \\
\left\langle P_{8}, P_{6} P_{2}, P_{4}^{2}, P_{4} P_{2}^{2}, P_{2}^{4}\right\rangle & \text { for } i=4, \\
\left\langle P_{10}, P_{8} P_{2}, P_{6} P_{4}, P_{6} P_{2}^{2}, P_{4}^{2} P_{2}, P_{4} P_{2}^{3}, P_{2}^{5}\right\rangle & \text { for } i=5 .\end{cases}
\end{aligned}
$$

It suffices to verify that, for $i=1, \ldots, 5$, the $d_{i}$ global sections given of $\left.\mathscr{O}(2 i)\right|_{\widetilde{M}}$ are linearly independent.

These are simple machine calculations. Starting with six $\mathbb{Q}$-rational points on $\mathbf{P}^{2}$ in general position, one may compute Coble's irrational invariants and obtain a point on $\widetilde{M} \subset \mathbf{P}^{39}$. Evaluating the power sums and the expressions listed yields a vector in $\mathbb{Q}^{d_{i}}$. Having repeated this process $N$ times, one ends up with a $d_{i} \times N$-matrix and the task is to show that it is of rank $d_{i}$. The calculation may be executed over the rationals or modulo a prime of moderate size. 
The linear maps res $_{i}$ are thus surjective and we actually found bases for $\Gamma\left(\widetilde{M},\left.\mathscr{O}(2 i)\right|_{\widetilde{M}}\right)^{W\left(E_{6}\right)}$, consisting of sections extending to the whole of $\mathbf{P}^{39}$. This is enough to prove assertion i).

Third step. The proofs of ii) and iii).

The rational map $\varphi$ is defined by five sections $s_{i} \in \Gamma\left(\widetilde{M},\left.\mathscr{O}(2 i)\right|_{\widetilde{M}}\right)^{W\left(E_{6}\right)}$, for $i=1, \ldots, 5$. To explicitly describe an extension to $\mathbf{P}^{39}$ as desired, the actual coefficients of $s_{1}, \ldots, s_{5}$ in the bases (3.5) have to be determined.

This is, in fact, an interpolation problem. Starting with a smooth cubic surface in the blown-up model, one may, as in the second step, directly compute the values of the 40 irrational invariants $\gamma$. and their power sums. On the other hand, using the methods described in A.1. Algorithm A.4, and A.6 it is typically possible to compute Clebsch's invariants $A, \ldots, E$. Having done this for sufficiently many surfaces, the 18 coefficients are fixed up to the appropriate scaling factors.

iii) is only a reformulation of ii).

Remarks 3.12. i) We find it quite noteworthy that the polynomials representing $s_{1}, \ldots, s_{5}$ may actually be chosen to be $S_{40}$-invariant, particularly in view of the fact that $W\left(E_{6}\right)$ is of an enormous index in $S_{40}$.

ii) At least for $i \geq 2$, the full restriction homomorphism

$$
\Gamma\left(\mathbf{P}^{39}, \mathscr{O}(2 i)\right) \longrightarrow \Gamma\left(\widetilde{M},\left.\mathscr{O}(2 i)\right|_{\widetilde{M}}\right)
$$

is surjective, as may be shown by the usual cohomological argument. Recall from Lemma 2.11,iii) that the Castelnuovo-Mumford regularity of $\mathscr{I}_{\widetilde{M}}$ is equal to 5 . Since $1+2 i \geq 5$, this implies $H^{1}\left(\mathbf{P}^{39}, \mathscr{I}_{\widetilde{M}}(2 i)\right)=0$ [27, Lecture 14]. Knowing this, the claim immediately follows.

iii) Since the appearance of the results of Clebsch and Coble, many mathematicians studied the moduli spaces $\widetilde{\mathscr{M}}$ and $\mathscr{M}$, as well as the canonical morphism pr: $\widetilde{\mathscr{M}} \rightarrow \mathscr{M}$ connecting them. We do not intend to give a complete list, as this would be a hopeless task.

But, in addition to the references given above, we feel that we should mention the article [7] of E. Colombo, B. van Geemen, and E. Looijenga, where the authors reinterpret Coble's results in terms of root systems. For us, some of their geometric results on the completions of the moduli spaces $\widetilde{\mathscr{M}}$ and $\mathscr{M}$ turned out to be helpful.

\section{Twisting Coble's gamma variety}

4.1. Fix a continuous homomorphism $\rho: \operatorname{Gal}(\bar{K} / K) \rightarrow W\left(E_{6}\right)$ and consider

$$
\begin{aligned}
& F_{\rho}:\{K \text {-schemes }\} \longrightarrow\{\text { sets }\}, \\
& S \mapsto\left\{\text { marked cubic surfaces over } S_{\bar{K}} \text { such that } \operatorname{Gal}(\bar{K} / K)\right. \\
&\text { operates on the } 27 \text { lines as described by } \rho\} / \sim,
\end{aligned}
$$

the moduli functor, twisted by $\rho$.

Theorem 4.2. The functor $F_{\rho}$ is representable by a K-scheme $\widetilde{\mathscr{M}}_{\rho}$ that is a twist of $\widetilde{\mathscr{M}}$.

Proof. Let $L / K$ be a finite Galois extension such that $\operatorname{Gal}(\bar{K} / L) \subseteq \operatorname{ker} \rho$. Then the restriction of $F_{\rho}$ to the category of $L$-schemes is clearly represented by the $L$-scheme $\widetilde{\mathscr{M}}_{L}:=\widetilde{\mathscr{M}} \times_{\text {Spec } K} \operatorname{Spec} L$.

For $g \in W\left(E_{6}\right)$, let $T_{g}: \widetilde{\mathscr{M}}_{L} \rightarrow \widetilde{\mathscr{M}}_{L}$ be the morphism corresponding to the operation of $g$ on the 27 labels. This is the base extension of a morphism $T_{g}^{K}: \widetilde{\mathscr{M}} \rightarrow \widetilde{\mathscr{M}}$. 
Further, for $\sigma \in \operatorname{Gal}(L / K)$, write $\sigma: \widetilde{\mathscr{M}}_{L} \rightarrow \widetilde{\mathscr{M}}_{L}$ for the morphism induced by $\sigma^{-1}: L \leftarrow L$. Then

$$
\begin{aligned}
\operatorname{Gal}(L / K) & \longrightarrow \operatorname{Mor}_{K}\left(\widetilde{\mathscr{M}}_{L}, \widetilde{\mathscr{M}}_{L}\right), \\
\sigma & \mapsto T_{\rho(\sigma)} \circ \sigma
\end{aligned}
$$

is a descent datum. Indeed, for $\sigma, \tau \in \operatorname{Gal}(L / K)$, one has

$$
T_{\rho(\sigma)} \circ \sigma \circ T_{\rho(\tau)} \circ \tau=T_{\rho(\sigma)} \circ\left(\sigma \circ T_{\rho(\tau)} \circ \sigma^{-1}\right) \circ \sigma \circ \tau=T_{\rho(\sigma)} \circ T_{\rho(\tau)} \circ \sigma \circ \tau=T_{\rho(\sigma \tau)} \circ \sigma \tau .
$$

Observe that $\sigma \circ T_{\rho(\tau)} \circ \sigma^{-1}=T_{\rho(\tau)}$, as $T_{\rho(\tau)}$ is the base extension of a $K$-morphism. Galois descent [32, Chapitre V, $\S 4, \mathrm{n}^{\circ} 20$, or 22, Proposition 2.5] yields a $K$-scheme $\mathscr{\mathscr { M }}_{\sigma}$ such that $\mathscr{\mathscr { M }}_{\sigma} \times_{\operatorname{Spec} K} \operatorname{Spec} L \cong \widetilde{\mathscr{M}}_{L}$.

By the universal property of the moduli scheme $\widetilde{\mathscr{M}}_{L}$, for every $K$-scheme $S$, the set $F_{\rho}(S)$ is in bijection with the set of all morphisms $S_{L} \rightarrow \widetilde{\mathscr{M}}_{L}$ of $L$-schemes such that, for every $\sigma \in \operatorname{Gal}(L / K)$, the diagram

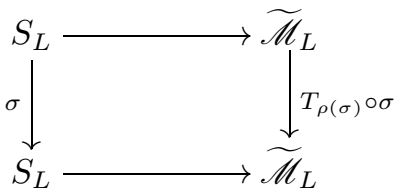

commutes. Galois descent for morphisms of schemes [22, Proposition 2.8] shows that this datum is equivalent to giving a morphism $S \rightarrow \widetilde{\mathscr{M}}_{\rho}$ of $K$-schemes.

4.3. This result suggests the following strategy to construct a smooth cubic surface $C$ over $\mathbb{Q}$ such that the Galois group $\operatorname{Gal}(\overline{\mathbb{Q}} / \mathbb{Q})$ acts upon the lines of $C$ via a prescribed subgroup $G \subseteq W\left(E_{6}\right)$.

Strategy. i) First, find a Galois extension $L / \mathbb{Q}$ such that $\operatorname{Gal}(L / \mathbb{Q}) \cong G$. This defines the homomorphism $\rho$.

ii) Then a $\mathbb{Q}$-rational point $P \in \widetilde{\mathscr{M}}_{\rho}(\mathbb{Q})$ is sought.

iii) For the corresponding cubic surface $\mathscr{C}_{P}$ over $\mathbb{Q}$, the Galois group $\operatorname{Gal}(\overline{\mathbb{Q}} / \mathbb{Q})$ operates on the 27 lines exactly as desired.

Unfortunately, we do not have the universal family over $\widetilde{\mathscr{M}}_{\rho}$ at our disposal, at least not in a sufficiently explicit form. Thus, given a rational point $P \in \widetilde{\mathscr{M}}_{\rho}(\mathbb{Q})$, only the 40 irrational invariants $\gamma$. will be known and the cubic surface has to be reconstructed from this information. But, anyway, searching for a $\mathbb{Q}$-rational point on $\widetilde{\mathscr{M}}_{\rho}$ will be our main task.

Remarks 4.4. i) There is the embedding $\gamma^{\prime}: \widetilde{\mathscr{M}}_{L} \hookrightarrow \mathbf{P}_{L}^{79}$, and both kinds of morphisms, $\sigma$ and $T_{\rho(\sigma)}$, easily extend to $\mathbf{P}_{L}^{79}$. One has

$$
\begin{aligned}
\sigma:\left(x_{0}: \ldots: x_{79}\right) & \mapsto\left(\sigma\left(x_{0}\right): \ldots: \sigma\left(x_{79}\right)\right) \\
T_{\rho(\sigma)}:\left(x_{0}: \ldots: x_{79}\right) & \mapsto\left(x_{\Pi(\rho(\sigma))^{-1}(0)}: \ldots: x_{\Pi(\rho(\sigma))^{-1}(79)}\right) .
\end{aligned}
$$

In the second formula, $\Pi: W\left(E_{6}\right) \hookrightarrow S_{80}$ is the permutation representation on the irrational invariants $\pm \gamma$. To explain why the inverses are to be taken, recall that $T_{\rho(\sigma)}$ permutes the irrational invariants, i.e. the coordinates. The element $x_{i}$ is moved to position $\Pi(\rho(\sigma))(i)$. Our formula describes exactly this procedure.

ii) To give a $K$-rational point on $\widetilde{\mathscr{M}}_{\rho}$ is thus equivalent to giving an $L$-rational point $\left(x_{0}: \ldots: x_{79}\right)$ on $\gamma^{\prime}\left(\mathscr{M}_{L}\right)$ such that

$$
\left(\sigma\left(x_{\Pi(\rho(\sigma))^{-1}(0)}\right): \ldots: \sigma\left(x_{\Pi(\rho(\sigma))^{-1}(79)}\right)\right)=\left(x_{0}: \ldots: x_{79}\right)
$$


or, equivalently, $\left(\sigma\left(x_{0}\right): \ldots: \sigma\left(x_{79}\right)\right)=\left(x_{\Pi(\rho(\sigma))(0)}: \ldots: x_{\Pi(\rho(\sigma))(79)}\right)$ for every $\sigma \in \operatorname{Gal}(L / K)$.

iii) The stronger condition that

$$
\left(\sigma\left(x_{\Pi(\rho(\sigma))^{-1}(0)}\right), \ldots, \sigma\left(x_{\Pi(\rho(\sigma))^{-1}(79)}\right)\right)=\left(x_{0}, \ldots, x_{79}\right)
$$

for all $\sigma \in \operatorname{Gal}(L / K)$ defines a descent datum for vector spaces and, hence, a 80-dimensional $K$-vector space in $L^{80}$.

Further, the linear relations between the irrational invariants $\pm \gamma$. are generated by such with coefficients in $K$. In fact, rational numbers are possible as coefficients. Hence, they form an $L$-vector space that is invariant under both operations, those of $\operatorname{Gal}(L / K)$ and of $W\left(E_{6}\right)$. This shows that the linear relations are respected by the descent datum. Galois descent yields a 10 -dimensional $K$-vector space $V$ in the 10 -dimensional $L$-vector space defined by the linear relations.

iv) Analogous observations hold for the space of cubic relations. They form a 30-dimensional $L$-vector space that is closed under the operations of $\operatorname{Gal}(L / K)$ and $W\left(E_{6}\right)$ and, therefore, respected by the descent datum. Descent yields a 30 -dimensional $K$-vector space.

Consequently, the Zariski closure of $\widetilde{\mathscr{M}}_{\rho} \subset \mathbf{P}(V) \cong \mathbf{P}_{K}^{9}$ is the intersection of 30 $K$-rational cubic hypersurfaces.

General remarks on our approach to explicit Galois descent.

4.5. i) Our approach works as soon as we are given a finite Galois extension $L / K$, a subscheme $M \subseteq \mathbf{P}_{L}^{N}$, and a $K$-linear operation $T$ of $G:=\operatorname{Gal}(L / K)$ on $\mathbf{P}_{L}^{N}$ such that $M$ is invariant under $T_{\sigma} \circ \sigma$ for every $\sigma \in G$. Linearity means that there is given a representation $A: G \rightarrow \mathrm{GL}_{N+1}(K)$ such that $T_{\sigma}$ is defined by the matrix $A(\sigma)$.

In fact, every representation of a finite group is a subrepresentation of a sum of several copies of the regular representation. Consequently, $M$ allows a linearly equivalent embedding into some $\mathbf{P}^{N^{\prime}}, N^{\prime} \geq N$, such that the $T_{\sigma}$ extend to $\mathbf{P}^{N^{\prime}}$ as automorphisms that simply permute the coordinates according to a permutation representation $\pi: G \rightarrow S_{N^{\prime}+1}$. We prefer permutations versus matrices in the description of the theory only in order to keep notation concise.

ii) Consider the particular case that the Galois descent is a twist, i.e., a $K$-scheme $M_{K}$ is given such that $M=M_{K} \times$ Spec $K \operatorname{Spec} L$ and the goal is to construct another $K$-scheme $M_{K}^{\prime}$ such that $M_{K}^{\prime} \times_{\text {Spec } K} \operatorname{Spec} L \cong M$.

Then the descent datum on $M$ is of the form $\left\{T_{\sigma} \circ \sigma\right\}_{\sigma \in G}$, where the $T_{\sigma}$ are in fact base extensions of $K$-scheme automorphisms of $M_{K}$. What is missing in order to apply i) is exactly a linearization of the operation $T: G \rightarrow \operatorname{Aut}(M)$.

iii) At least in principle, such a linearization always exists as soon as $M_{K}$ is quasiprojective. Indeed, let $\mathscr{L} \in \operatorname{Pic}\left(M_{K}\right)$ be a very ample invertible sheaf. Then $G$ operates $\mathscr{O}_{M_{K}}$-linearly on the very ample invertible sheaf $\bigotimes_{g \in G} T_{g}^{*} \mathscr{L}$. Use its global
sections for a projective embedding.

\section{An application to the inverse Galois problem for CUbic surfaces}

A general algorithm.

Algorithm 5.1 (Cubic surface for a given group). Given a subgroup $G \subseteq W\left(E_{6}\right)$ and a field such that $\operatorname{Gal}(L / \mathbb{Q}) \cong G$, this algorithm computes a smooth cubic surface $C$ over $\mathbb{Q}$ such that $\operatorname{Gal}(\overline{\mathbb{Q}} / \mathbb{Q})$ operates upon the lines of $C$ via the group $\operatorname{Gal}(L / \mathbb{Q})$. 
i) Fix a system $\Gamma \subseteq G$ of generators of $G$. For every $g \in \Gamma$, store the permutation $\Pi(g) \in S_{80}$, which describes the operation of $g$ on the 80 irrational invariants $\pm \gamma$. Further fix, once and for ever, ten of the $\pm \gamma$. that are linearly independent. Express the other 70 explicitly as linear combinations of these basis vectors.

ii) For every $g \in \Gamma$, determine the $10 \times 10$-matrix describing the operation of $g$ on the 10-dimensional $L$-vector space $\langle\gamma$. $\rangle$. Use the explicit basis, fixed in i).

iii) Choose an explicit basis of the field $L$ as a $\mathbb{Q}$-vector space. Finally, make explicit the isomorphism $\rho^{-1}: G \rightarrow \operatorname{Gal}(L / \mathbb{Q}) \subseteq \operatorname{Hom}_{\mathbb{Q}}(L, L)$, i.e., write down a matrix for every $g \in \Gamma$.

iv) Now, the condition that

$$
\left(\sigma\left(x_{\Pi(\rho(\sigma))^{-1}(0)}\right), \ldots, \sigma\left(x_{\Pi(\rho(\sigma))^{-1}(79)}\right)\right)=\left(x_{0}, \ldots, x_{79}\right)
$$

for all $\sigma \in \operatorname{Gal}(L / \mathbb{Q})$ is an explicit $\mathbb{Q}$-linear system of equations in $10[L: \mathbb{Q}]$ variables. In fact, we start with $\Gamma$ instead of $\operatorname{Gal}(L / \mathbb{Q})$ and get $80[L: \mathbb{Q}] \# \Gamma$ equations. The result is a ten-dimensional $\mathbb{Q}$-vector space $V \subset\langle\gamma$. $\rangle$, described by an explicit basis.

v) Convert the 30 cubic forms defining the image of $\gamma_{L}: \widetilde{\mathscr{M}}_{L} \hookrightarrow \mathbf{P}_{L}^{79}$ into terms of this basis of $V$. The result is 30 explicit cubic forms with coefficients in $\mathbb{Q}$. They describe the Zariski closure of $\widetilde{\mathscr{M}}_{\rho}$ in $\mathbf{P}(V)$.

vi) Search for a $\mathbb{Q}$-rational point on this variety.

vii) From the coordinates of the point found, read the 40 irrational invariants $\gamma$. Then use formulas (3.4) in order to calculate Clebsch's invariants $A, \ldots, E$. Finally, solve the equation problem as described in A.8 and Algorithm A.10.

In the case that $\mathrm{A.8}$ or Algorithm A.10 fails, return to step vi)

Remarks 5.2. i) An important implementation trick was the following. We do not solve the linear system of equations in $L^{10}$ but in $\mathscr{O}_{L}^{10}$, for $\mathscr{O}_{L} \subset L$ the maximal order. The result is then a rank-10 $\mathbb{Z}$-lattice. Via the Minkowski embedding, this carries a scalar product. Thus, it may be reduced using the LLL-algorithm 25. It turned out in practice that points of very small height occur when taking the LLL-basis for a projective coordinate system.

Applying the LLL-algorithm to the lattice constructed from the maximal order should be considered as a first step towards a multivariate polynomial reduction and minimization algorithm for noncomplete intersections.

ii) There are two points where Algorithm 5.1 may possibly fail. First, it may happen that no $\mathbb{Q}$-rational point is found on $\widetilde{\mathscr{M}}_{\rho}$. Then one has to start with a different field having the same Galois group.

Second, A.8 or Algorithm A.10 may fail, because of $E=0, \Delta=0$, or $F=0$; cf. Remarks A.12 ii) and iii). This means that the cubic surface found either has no proper pentahedron, or is singular, or has nontrivial automorphisms.

These cases exclude a divisor from the compactified moduli space $\mathbf{P}(1,2,3,4,5)$. Thus, Algorithm 5.1 works generically. In our experiments to construct examples for the remaining conjugacy classes, we met the situation that $\Delta=0$, but not the situations that $E=0$ or $F=0$.

iii) In order to get number fields with a prescribed Galois group, we used J. Klüners' number field database http://galoisdb.math.upb.de .

The 51 remaining conjugacy classes.

Remark 5.3 (Previous examples). There are exactly 350 conjugacy classes of subgroups in $W\left(E_{6}\right)$. For a generic cubic surface, the full $W\left(E_{6}\right)$ acts upon the lines. 
In previous articles, we presented constructions producing examples for the index two subgroup $D^{1} W\left(E_{6}\right)$ [11, all subgroups stabilizing a double-six [13, all subgroups stabilizing a pair of Steiner trihedra [14, and all subgroups stabilizing a line [15.

There are 158 conjugacy classes stabilizing a double-six, 63 conjugacy classes stabilizing a pair of Steiner trihedra but no double-six, and 76 conjugacy classes stabilizing a line but neither a double-six nor a pair of Steiner trihedra. Summing up, the previous constructions completed 299 of the 350 conjugacy classes of subgroups.

5.4. For some of the 51 conjugacy classes not yet covered, cubic surfaces are easily constructed. In fact,

i) there are the twists of the diagonal surface

$$
X_{0}^{3}+X_{1}^{3}+X_{2}^{3}+X_{3}^{3}=0 .
$$

These cubic surfaces may be written as $\operatorname{Tr}_{A / \mathbb{Q}} a l^{3}=0$ for $A$ an étale algebra of degree 4 over $\mathbb{Q}, a \in A$, and $l$ a linear form in four variables over $A$. They have 18 Eckardt points [10, section 9.1.4].

This approach yields nine of the 51 remaining conjugacy classes. Their numbers in the list are $245,246,289,301,303,327,337,338$, and 346.

ii) The surfaces of the type

$$
\lambda X_{0}^{3}=F_{3}\left(X_{1}, X_{2}, X_{3}\right)
$$

generically have nine Eckardt points, the nine inflection points of the cubic curve, given by $F_{3}\left(X_{1}, X_{2}, X_{3}\right)=0$. This approach yields another seven conjugacy classes. Their numbers are 172, 235, 236, 299, 317, 332, and 345 .

Remark 5.5. In these cases, the sets of Eckardt points are Galois invariant. Hence, these two constructions produce Galois groups that are contained in the stabilizers of these sets. These are the two maximal subgroups of index 40. On the other hand, the field of definition of the 27 lines contains $\zeta_{3}$, essentially due to the Weil pairing on the relevant elliptic curve. Thus, there is no hope to construct in this way examples for all the groups contained in these two maximal subgroups.

5.6. Further, there are a few obvious ways to try a computational brute force attack.

i) We systematically searched through the cubic surfaces such that all 20 coefficients are in the range $\{-1,0,1\}$. This led to examples for 14 more conjugacy classes. They correspond to the numbers 144, 232, 267, 269, 272, 273, 305, $307,309,310,329,333,334$, and 339 in the list.

ii) Similarly, but less systematically, we searched for cubic surfaces with a rational tritangent plane but no rational line. This means to choose a cubic field extension $K / \mathbb{Q}$ with splitting field of type $A_{3}$ or $S_{3}$, to fix a linear form $l \in K\left[X_{1}, X_{2}, X_{3}\right]$, and to search for surfaces of the type

$$
\mathrm{N}_{K / \mathbb{Q}} l+X_{0} F_{2}\left(X_{0}, X_{1}, X_{2}, X_{3}\right)=0 .
$$

As there are only ten unknown coefficients, we could search in a little wider range. Note that the generic case of this construction gives the remaining maximal subgroup of index 45 in $W\left(E_{6}\right)$.

Six surfaces with orbit structures of types $[3,12,12]$ and $[3,24]$ have been found. The corresponding gap numbers are 90, 153, 260, 324, 335, and 344 . 
iii) In analogy with i), we searched through all pentahedral equations with small coefficients. As this family has only 5 parameters, we could inspect all surfaces with coefficients up to 500. Similarly, we inspected all pentahedral equations with unit fractions as coefficients and a denominator not more than 500. This was motivated by simplifications shown in [12, Fact 2.8].

This approach results in examples for group № 149 of order 24 and № 326 of order 324. The pentahedral coefficients are $\left[\frac{1}{256}, \frac{1}{241}, \frac{1}{225}, \frac{1}{81}, \frac{1}{81}\right]$ and $\left[\frac{1}{84}, \frac{1}{64}, \frac{1}{52}, \frac{1}{49}, 1\right]$.

iv) Following the same path, we systematically searched through all invariant vectors $[A, \ldots, E]$ such that $|A|, \ldots,|E|<100$. In each case, we solved the equation problem as described in A.8 and Algorithm A.10. This led to examples for six more conjugacy classes. Their gap numbers are 216, 239, 302, 313, 319, and 336 .

Remark 5.7 (Concerning approach i)). A priori, the search through the surfaces with small coefficients, as described in i), requires the inspection of more than $3 \cdot 10^{9}$ surfaces. However, using symmetry, we can do much better. For this, one has to enumerate the $3^{12}$ possible combinations of monomials of the form $X_{0}^{2} X_{1}, \ldots, X_{2} X_{3}^{2}$. Then one may split this set into orbits under the operation of $(\mathbb{Z} / 2 \mathbb{Z})^{4} \rtimes S_{4}$, where $S_{4}$ permutes the four indeterminates and $(\mathbb{Z} / 2 \mathbb{Z})^{4}$ changes their signs.

This leads to 1764 representatives. Each representative can be extended to a cubic surface in $3^{8}$ ways by choosing coefficients for the monomials $X_{0}^{3}, X_{1}^{3}, X_{2}^{3}$, $X_{3}^{3}, X_{0} X_{1} X_{2}, X_{0} X_{1} X_{3}, X_{0} X_{2} X_{3}$, and $X_{1} X_{2} X_{3}$. Thus, approximately $1.1 \cdot 10^{7}$ surfaces had to be inspected.

Remark 5.8 (Concerning approaches iii) and iv)). Before trying approaches iii) and iv), exactly 15 conjugacy classes were left open. It turned out that all these were either even, i.e. contained in the index-2 subgroup $D^{1} W\left(E_{6}\right) \subset W\left(E_{6}\right)$, or had a factor commutator group that was cyclic of order 4 or 8 . This implies strong restrictions on the discriminant $\Delta$ of the cubic surfaces sought.

To understand this, recall the following property, which partly characterizes the discriminant $\Delta$. If the 27 lines on a smooth cubic surface $C$ over $\mathbb{Q}$ are acted upon by an odd Galois group $G \subseteq W\left(E_{6}\right)$, then the quadratic number field corresponding to the subgroup $G \cap D^{1} W\left(E_{6}\right) \subset G$ is exactly $\mathbb{Q}(\sqrt{(-3) \Delta})$ [11, Theorem 2.12]. Correspondingly, if $G \subseteq W\left(E_{6}\right)$ is even, then $(-3) \Delta$ must be a perfect square.

In the odd case, the factor commutator group $G / D^{1} G$ of $G$ surjects onto $G / G \cap$ $D^{1} W\left(E_{6}\right) \cong \mathbb{Z} / 2 \mathbb{Z}$. Hence, $G / D^{1} G$ corresponds to a subfield $L$ of the field of definition of the 27 lines containing $\mathbb{Q}(\sqrt{(-3) \Delta})$.

In other words, there is an embedding $\mathbb{Q}(\sqrt{(-3) \Delta}) \subset L$ into a field $L$ that is Galois and cyclic of degree 4 (or even 8 ) over $\mathbb{Q}$.

Lemma 5.9. i) If a quadratic number field $\mathbb{Q}(\sqrt{D})$ allows an embedding into a field $L$ that is Galois and cyclic of degree 4 over $\mathbb{Q}$, then $D>0$ and all prime factors $p \equiv 3(\bmod 4)$ in $D$ have an even exponent.

ii) If $\mathbb{Q}(\sqrt{D})$ even allows an embedding into a field Galois and cyclic of degree 8, then the same is true for all primes $p \equiv 5(\bmod 8)$.

Proof. i) is shown in [33, Theorem 1.2.4]. For ii), the proof is analogous. Both results are direct applications of class field theory.

We used this restriction in approaches iii) and iv) as a highly efficient pretest. It immediately ruled out most of the candidates. 
5.10. To summarize, using relatively naive methods, we found examples for 44 of the 51 remaining conjugacy classes. Thus, only for the last seven, we had to use the main algorithm. In the list, they correspond to the numbers $73,155,169,177$, 179,266 , and 286 .

Remarks concerning the running times.

5.11. We implemented the main algorithm and the elementary algorithms described in the appendix in magma, version 2.18. We worked on one core of an Intel $^{(\mathrm{R})} \mathrm{Core}^{(\mathrm{TM})} 2$ Duo E8300 processor.

i) To compute the numerical invariants of the gamma variety $\widetilde{M}$, given in Lemma 2.11, the running times were less than 0.1 seconds.

ii) To determine the coefficients in Theorem 3.9 ii), the running time was around 10 seconds per knot.

There are certainly faster methods to compute Clebsch's invariants for a given cubic surface. We preferred the approach described, as it does not depend on deep theory and leads to a compact code. In fact, we do much more than just calculating Clebsch's invariants, as we completely determine the pentahedron.

iii) Our code implementing the main algorithm for the subgroup № 73 , which is cyclic of order nine, is available on both author's web pages as a file named c9_example.m. It runs within a few seconds on the magma online calculator.

As one might expect, it takes longer to run examples that involve larger number fields. Further, for the point search, a completely naive $O\left(N^{10}\right)$-algorithm is used. Thus, the existence of a point of very small height is absolutely necessary for our implementation to succeed.

\section{Appendix A. Some ELEMEntary Algorithms}

Computing an equation from six blow-up points.

A.1. Given six points $p_{1}, \ldots, p_{6} \in \mathbf{P}^{2}(K)$ in general position, it is pure linear algebra to compute a sequence of 20 coefficients for the corresponding cubic surface. First, one has to determine a base of the kernel of a $6 \times 10$-matrix in order to find four linearly independent cubic forms $F_{1}, \ldots, F_{4}$ vanishing in $p_{1}, \ldots, p_{6}$. To find the cubic relation between $F_{1}, \ldots, F_{4}$ means to solve a highly overdetermined homogeneous linear system of 220 equations in 20 variables.

Remark A.2. Actually, there is a second algorithm, which is simpler but certainly less standard. Starting with the six points $p_{1}, \ldots, p_{6} \in \mathbf{P}^{2}(K)$, one may use formula (85) of A. B. Coble [5] to find hexahedral coefficients $a_{0}, \ldots, a_{5} \in K$ for the corresponding cubic surface. From this, an explicit equation is immediately obtained.

Computing the pentahedron and Clebsch's invariants from an equation.

A.3. For a cubic surface in pentahedral form,

$C\left(X_{0}, X_{1}, X_{2}, X_{3}\right):=a_{0} X_{0}^{3}+a_{1} X_{1}^{3}+a_{2} X_{2}^{3}+a_{3} X_{3}^{3}-a_{4}\left(X_{0}+X_{1}+X_{2}+X_{3}\right)^{3}=0$

such that $a_{0}, \ldots, a_{4} \in K \backslash\{0\}$, its Hessian det $\frac{\partial^{2} C}{\partial X_{i} \partial X_{j}}\left(X_{0}, X_{1}, X_{2}, X_{3}\right)=0$ has exactly ten singular points. These are simply the intersection points of three of the five planes defined by $X_{0}=0, \ldots, X_{3}=0$ and $X_{4}:=-\left(X_{0}+X_{1}+X_{2}+X_{3}\right)=0$. Thus, each plane contains six of the ten singular points. 
Hence, given a cubic surface in the form of a sequence of 20 coefficients, one has to compute its Hessian first. If the singular points have a configuration different from what was described, then there is no pentahedron. Otherwise, one has to determine the five planes through six singular points and to normalize the corresponding linear forms $l_{0}, \ldots, l_{4}$ such that their sum is zero. To find the five coefficients $a_{0}, \ldots, a_{4}$ means to solve an overdetermined homogeneous linear system of 20 equations in five variables.

There is, however, one serious practical difficulty. The pentahedron is typically defined only over an $S_{5}$-extension of the base field $K$. For this situation, we have the following algorithm.

Algorithm A.4 (Pentahedron from a cubic surface). Let a cubic surface $C$ be given as a sequence of 20 coefficients. Suppose that there is a proper pentahedron and that its field of definition is an $S_{5^{-}}$or $A_{5^{-}}$extension of the base field $K$. Then this algorithm computes the pentahedral form.

i) Determine a Gröbner basis for the ideal $\mathscr{I}_{H_{\text {sing }}} \subset K\left[X_{0}, \ldots, X_{3}\right]$ of the singular locus of the Hessian $H$ of $C$. In particular, this yields a univariate degree-10 polynomial $\bar{F}$ defining the $S_{5^{-}}$or $A_{5}$-extension.

ii) Uncover a degree-5 polynomial $F$ with the same splitting field. When $K=$ $\mathbb{Q}$, this may be done as follows. Run a variant of Stauduhar's algorithm 35. This yields $p$-adic approximations of the ten zeroes of $\bar{F}$ together with an explicit description of the operation of $S_{5}$ or $A_{5}$. Then calculate $p$-adically a relative resolvent polynomial [35. Theorem 4], corresponding to the inclusion $S_{4} \subset S_{5}$ or $A_{4} \subset A_{5}$, respectively. From this, the polynomial $F \in \mathbb{Q}[T]$ is obtained by rational recovery.

Put $L$ to be the extension field defined by $F$. Clearly, $[L: K]=5$.

iii) Factorize $\bar{F}$ over $L$. Two irreducible factors, $\bar{F}_{1}$ of degree 4 and $\bar{F}_{2}$ of degree 6 , are found.

iv) Determine, in a second Gröbner base calculation, an element of minimal degree in the ideal $\left(\mathscr{I}_{H_{\text {sing }}}, \bar{F}_{2}\right) \subset L\left[X_{0}, \ldots, X_{3}\right]$. The result is a linear polynomial $l$. Its conjugates define the five individual planes that form the pentahedron.

v) Scale $l$ by a suitable nonzero factor from $L$ such that $\operatorname{Tr}_{L / K} l=0$. This amounts to solving over $K$ a homogeneous system of four linear equations in five variables. Then calculate $a \in L$ such that the equation of the surface is exactly $\operatorname{Tr}_{L / K} a l^{3}=0$.

Return $a$. Its five conjugates are the pentahedral coefficients of $C$. One might want to return $l$ as a second value.

Remarks A.5. i) Observe that it is not necessary to perform any computations in the Galois hull $\widetilde{L}$ of $L$.

ii) Let us explain the idea behind Algorithm A.4. The Galois group $\operatorname{Gal}(\widetilde{L} / K)$ $\cong S_{5}$ or $A_{5}$ permutes the five planes of the pentahedron. The ten singular points of the Hessian are in bijection with sets of three planes and permuted accordingly. Further, $\operatorname{Gal}(\widetilde{L} / L)$ is the stabilizer of one plane. Under this group, the six singular points that lie on that plane form an orbit and the four others form another.

The same is still true after projection to the $\left(X_{0}, X_{1}\right)$-line. Indeed, the Galois operation immediately carries over to the coordinates. Further, no two of the ten points may coincide after projection, as this would define a nontrivial block structure for the image of $\operatorname{Gal}(\widetilde{L} / K)$ in $S_{10}$. Our assumptions ensure, however, that this subgroup is primitive. This explains the type of factorization described in step iii). 
In addition, $\left(\mathscr{I}_{H_{\text {sing }}}, \bar{F}_{2}\right)$ is the ideal of the six singular points lying on the $L$-rational plane. That is why a Gröbner base calculation for this ideal may discover the equation for that plane.

iii) It is not necessary to check the assumptions of this algorithm in advance, as its output may be verified by a direct calculation. Actually, when there is no proper pentahedron, the algorithm should usually fail in the very first step, detecting that $K\left[X_{0}, \ldots, X_{3}\right] / \mathscr{I}_{H_{\text {sing }}}$ is not of length ten. If the Galois group is too small, then more than two irreducible factors or even multiple factors may occur in step iii).

iv) It would certainly be possible to make Algorithm A.4 work for an arbitrary subgroup of $S_{5}$. Somewhat paradoxically, for small subgroups, the algorithm should be of lower complexity but harder to describe. We did not work out the details, since the present version turned out to be sufficient for our purposes.

v) To compute the pentahedron for a cubic surface given by an explicit equation was considered as being a hopeless task before the formation of modern computer algebra. The reader might compare the concluding remarks of [23, section 6.6.2].

A.6. Clebsch's invariants from pentahedral coefficients. Having found the pentahedral coefficients, Clebsch's invariants may be directly calculated using formulas 3.2 .

Remark A.7. The algorithms described up to this point were used in the proof of Theorem 3.9 ii).

Computing a cubic surface from Clebsch's invariants. The equation problem.

A.8. The other way around, given Clebsch's invariants $[A, B, C, D, E]$ such that $E \neq 0$, one can calculate the corresponding base point in the pentahedral family as follows.

Replace $[A, B, C, D, E]$ by $\left[A^{\prime}, B^{\prime}, C^{\prime}, D^{\prime}, E^{\prime}\right]:=\left[A E^{3}, B E^{6}, C E^{9}, D E^{12}, E^{16}\right]$ and set $\sigma_{5}:=E^{2}$ first. Then put $\sigma_{1}:=\frac{B^{\prime}}{\sigma_{5}^{3}}, \sigma_{2}:=\frac{D^{\prime}}{\sigma_{5}^{6}}, \sigma_{4}:=\frac{C^{\prime}}{\sigma_{5}^{4}}$, and, finally, $\sigma_{3}:=\frac{\sigma_{4}^{2}-A^{\prime}}{4 \sigma_{5}}$. This may be simplified to

$$
\left[\sigma_{1}, \ldots, \sigma_{5}\right]=\left[B, D, \frac{C^{2}-A E}{4}, C E, E^{2}\right] .
$$

Remark A.9. If $\sigma_{1}, \ldots, \sigma_{5} \in K$, then one would strongly expect that the corresponding cubic surface is defined over $K$. We learn, however, from formulas (3.1) that $\mathscr{C}_{\left(\sigma_{1}, \ldots, \sigma_{5}\right)}$ is a priori defined only over the splitting field $L$ of the polynomial $g(T):=T^{5}-\sigma_{1} T^{4} \pm \ldots-\sigma_{5} \in K[T]$.

But, at least when $g$ has no multiple zeroes, $\mathscr{C}_{\left(\sigma_{1}, \ldots, \sigma_{5}\right)}$ is equipped with a canonical descent datum. Indeed, let $a_{0}, \ldots, a_{4} \in L$ be the zeroes of $g$. For $\sigma \in \operatorname{Gal}(L / K)$, denote by $\pi(\sigma) \in S_{5}$ the corresponding permutation of $a_{0}, \ldots, a_{4}$, i.e., $a_{\pi(\sigma)(i)}=$ $\sigma\left(a_{i}\right)$. Then put

$$
\begin{aligned}
\operatorname{Gal}(L / K) & \longrightarrow \operatorname{Mor}_{K}\left(\mathscr{C}_{\left(\sigma_{1}, \ldots, \sigma_{5}\right)}, \mathscr{C}_{\left(\sigma_{1}, \ldots, \sigma_{5}\right)}\right), \\
\sigma & \mapsto\left(\left(x_{0}: \ldots: x_{4}\right) \mapsto\left(\sigma\left(x_{\pi(\sigma)^{-1}(0)}\right): \ldots: \sigma\left(x_{\pi(\sigma)^{-1}(4)}\right)\right)\right) .
\end{aligned}
$$

It is easily checked that these morphisms indeed map $\mathscr{C}_{\left(\sigma_{1}, \ldots, \sigma_{5}\right)}$ onto itself and that they form a group operation.

Algorithm A.10 (Computation of the Galois descent). Given a separable polynomial $g(T)=T^{5}-\sigma_{1} T^{4} \pm \ldots-\sigma_{5} \in K[T]$ of degree five, this algorithm computes the Galois descent to $K$ of the cubic surface $\mathscr{C}_{\left(\sigma_{1}, \ldots, \sigma_{5}\right)}$. 
i) The polynomial $g$ defines an étale $K$-algebra $A:=K[T] /(g)$. Compute, according to the definition, the traces $t_{i}:=\operatorname{tr}_{A / K} T^{i}$ for $i=0, \ldots, 4$.

ii) Determine the kernel of the $1 \times 5$-matrix

$$
\left(\begin{array}{lllll}
t_{0} & t_{1} & t_{2} & t_{3} & t_{4}
\end{array}\right) \text {. }
$$

Choose linearly independent kernel vectors $\left(c_{i}^{(0)}, \ldots, c_{i}^{(4)}\right) \in K^{5}$ for $i=0, \ldots, 3$.

iii) Compute the term

$$
T \cdot\left[\sum_{j=0}^{4}\left(c_{0}^{(j)} X_{0}+\ldots+c_{3}^{(j)} X_{3}\right) T^{j}\right]^{3}
$$

modulo $g(T)$. This is a cubic form in $X_{0}, \ldots, X_{3}$ with coefficients in $A$.

iv) Finally, apply the trace coefficient-wise and output the resulting cubic form in $x_{0}, \ldots, x_{3}$ with 20 rational coefficients.

Lemma A.11. For $g=T^{5}-\sigma_{1} T^{4} \pm \ldots-\sigma_{5} \in K[T]$ a separable polynomial, Algorithm A.10 computes a cubic surface over $K$ that is geometrically isomorphic to $\mathscr{C}_{\left(\sigma_{1}, \ldots, \sigma_{5}\right)}$.

Proof. The étale algebra $A=K[T] /(g)$ has five embeddings $i_{0}, \ldots, i_{4}: A \hookrightarrow \bar{K}$ into the algebraic closure. For $a_{0}, \ldots, a_{4} \in \bar{K}$ the images of $T$, we substituted into the equation

$$
a_{0} W_{0}+\ldots+a_{3} W_{3}+a_{4}\left(-W_{0}-\ldots-W_{3}\right)=0
$$

the linear form

$$
l_{0}:=C_{0} X_{0}+\ldots+C_{3} X_{3}=i_{0}\left(\sum_{j=0}^{4} c_{0}^{(j)} T^{j}\right) X_{0}+\ldots+i_{0}\left(\sum_{j=0}^{4} c_{3}^{(j)} T^{j}\right) X_{3}
$$

and $l_{1}, l_{2}, l_{3}$, three of its conjugates.

By construction, $C_{0}, \ldots, C_{3}$ form a basis of the $K$-vector space $N \subset A$ consisting of the elements of trace zero. In particular, $l_{4}:=-l_{0}-\ldots-l_{3}$ is indeed the fourth conjugate.

To show the isomorphy, we only need to ensure that $l_{0}, \ldots, l_{3}$ are linearly independent linear forms. This means that the $5 \times 4$-matrix $\left(C_{i}^{\sigma_{j}}\right)_{0 \leq j \leq 4,0 \leq i \leq 3}$ is of rank 4 . Extending $\left\{C_{0}, \ldots, C_{3}\right\}$ to a base $\left\{C_{0}, \ldots, C_{4}\right\}$ of $L$, it suffices to verify that the $5 \times 5$-matrix $\left(C_{i}^{\sigma_{j}}\right)_{0 \leq j \leq 4,0 \leq i \leq 4}$ has full rank. This is, however, independent of the choice of the base and clear for $C_{i}=T^{i}$. Indeed, we then have a Vandermonde matrix of determinant $\pm \prod_{i<j}\left(T^{\sigma_{i}}-T^{\sigma_{j}}\right)= \pm \prod_{i<j}\left(a_{i}-a_{j}\right) \neq 0$.

Remarks A.12. i) It is not hard to show that Algorithm A.10 computes the descent of the cubic surface $\mathscr{C}_{\left(\sigma_{1}, \ldots, \sigma_{5}\right)}$ according to exactly the descent data described above. We skip the proof as it closely follows the lines of [13, Theorem 6.6].

ii) Algorithm A.10 fails when $g$ has multiple zeroes. For the cubic surface $C$, this means that some of its pentahedral coefficients coincide. By [10, Example 9.1.25], this is equivalent to $C$ having an Eckardt point, which, in turn, means that $C$ has a nontrivial automorphism [10, Theorem 9.5.8]. Further, there is the well-known section $F \in \Gamma(\mathbf{P}(1,2,3,4,5), \mathscr{O}(25))$ that vanishes exactly on the locus corresponding to the cubic surfaces having an Eckardt point. In pentahedral coefficients, $F$ is given by the expression $I_{100}^{2}$ [10, section 9.4.5]. 
If $F=0$, then we actually face an ill-posed problem. Due to the presence of twists, the Clebsch invariants do not determine the cubic surface up to isomorphism over $K$, but only up to isomorphism over the algebraic closure $\bar{K}$. Thus, the information available to us is insufficient on principle in order to perform a Galois descent.

iii) Observe that, when $E \neq 0$ and $F \neq 0$, the discriminant $\Delta$ may nevertheless vanish. Then the corresponding cubic surface is singular.

A.13. It is classically called the equation problem [21, Definition 4.1.17] to determine an equation for the cubic surface when the invariants $A, \ldots, E$ are known. If $E \neq 0$ and $F \neq 0$, then A.8 and Algorithm A.10 together provide an algorithmic solution to the equation problem.

Remark A.14. If $F \neq 0$ but $E=0$, then one might start with $E=\varepsilon^{8} \in K[\varepsilon]$ (or $E=\varepsilon$ ) instead and run Algorithm A.10 over the function field. Unfortunately, the resulting cubic surface typically has bad reduction at $\varepsilon=0$. Thus, one cannot specialize $\varepsilon$ to 0 , naively. An application of J. Kollár's polynomial minimization algorithm [24, in particular Proposition 6.4.2] is necessary to find a good model. The reduction at $\varepsilon=0$ then solves the equation problem.

\section{ACKNOWLEDGEMENT}

We wish to thank Claus Fieker for several hints on how to handle in magma the relatively large number fields that occurred in the computations related to this project.

\section{REFERENCES}

[1] Daniel Allcock and Eberhard Freitag, Cubic surfaces and Borcherds products, Comment. Math. Helv. 77 (2002), no. 2, 270-296, DOI 10.1007/s00014-002-8340-4. MR.1915042 (2004c:14067)

[2] Arnaud Beauville, Complex algebraic surfaces, London Mathematical Society Lecture Note Series, vol. 68, Cambridge University Press, Cambridge, 1983. Translated from the French by R. Barlow, N. I. Shepherd-Barron and M. Reid. MR732439 (85a:14024)

[3] A. Cayley, On the triple tangent planes of surfaces of the third order, Cambridge and Dublin Mathematical Journal 4 (1849), 118-132.

[4] A. Clebsch, Ueber eine Transformation der homogenen Functionen dritter Ordnung mit vier Veränderlichen, J. für die Reine und Angew. Math. 58 (1861), 109-126.

[5] Arthur B. Coble, Point sets and allied Cremona groups. I, Trans. Amer. Math. Soc. 16 (1915), no. 2, 155-198, DOI 10.2307/1988716. MR.1501008

[6] Arthur B. Coble, Point sets and allied Cremona groups. III, Trans. Amer. Math. Soc. 18 (1917), no. 3, 331-372, DOI 10.2307/1988959. MR1501073

[7] Elisabetta Colombo, Bert van Geemen, and Eduard Looijenga, Del Pezzo moduli via root systems, Algebra, arithmetic, and geometry: in honor of Yu. I. Manin. Vol. I, Progr. Math., vol. 269, Birkhäuser Boston Inc., Boston, MA, 2009, pp. 291-337, DOI 10.1007/978-0-81764745-2_7. MR2641175(2011g:14086)

[8] Wolfram Decker and David Eisenbud, Sheaf algorithms using the exterior algebra, Computations in algebraic geometry with Macaulay 2, Algorithms Comput. Math., vol. 8, Springer, Berlin, 2002, pp. 215-249. MR 1949553

[9] Harm Derksen and Gregor Kemper, Computational invariant theory, Invariant Theory and Algebraic Transformation Groups, I, Springer-Verlag, Berlin, 2002. Encyclopaedia of Mathematical Sciences, 130. MR 1918599 (2003g:13004)

[10] Igor V. Dolgachev, Classical algebraic geometry, Cambridge University Press, Cambridge, 2012. A modern view. MR2964027

[11] Andreas-Stephan Elsenhans and Jörg Jahnel, The discriminant of a cubic surface, Geom. Dedicata 159 (2012), 29-40, DOI 10.1007/s10711-011-9643-7. MR2944518 
[12] Andreas-Stephan Elsenhans and Jörg Jahnel, On the arithmetic of the discriminant for cubic surfaces, J. Ramanujan Math. Soc. 27 (2012), no. 3, 355-373. MR2987232

[13] Andreas-Stephan Elsenhans and Jörg Jahnel, Cubic surfaces with a Galois invariant double-six, Cent. Eur. J. Math. 8 (2010), no. 4, 646-661, DOI 10.2478/s11533-010-0036-1. MR2671217(2011e:14045)

[14] Andreas-Stephan Elsenhans and Jörg Jahnel, Cubic surfaces with a Galois invariant pair of Steiner trihedra, Int. J. Number Theory 7 (2011), no. 4, 947-970, DOI 10.1142/S1793042111004253. MR2812646(2012f:11119)

[15] Andreas-Stephan Elsenhans and Jörg Jahnel, On cubic surfaces with a rational line, Arch. Math. (Basel) 98 (2012), no. 3, 229-234, DOI 10.1007/s00013-012-0356-4. MR2897445

[16] Andreas-Stephan Elsenhans and Jörg Jahnel, On the order three Brauer classes for cubic surfaces, Cent. Eur. J. Math. 10 (2012), no. 3, 903-926, DOI 10.2478/s11533-012-0042-6. MR2902222

[17] William Fulton, Introduction to toric varieties, Annals of Mathematics Studies, vol. 131, Princeton University Press, Princeton, NJ, 1993. The William H. Roever Lectures in Geometry. MR 1234037 (94g:14028)

[18] Bert van Geemen, A linear system on Naruki's moduli space of marked cubic surfaces, Internat. J. Math. 13 (2002), no. 2, 183-208, DOI 10.1142/S0129167X0200123X. MR1891207 (2002m:14028)

[19] A. Grothendieck and J. Dieudonné, Étude cohomologique des faisceaux cohérents (EGA III), Publ. Math. IHES 11 (1961), 17 (1963).

[20] Robin Hartshorne, Algebraic geometry, Springer-Verlag, New York, 1977. Graduate Texts in Mathematics, No. 52. MR0463157 (57 \#3116)

[21] Bruce Hunt, The geometry of some special arithmetic quotients, Lecture Notes in Mathematics, vol. 1637, Springer-Verlag, Berlin, 1996. MR1438547 (98c:14033)

[22] J. Jahnel, The Brauer-Severi variety associated with a central simple algebra, Linear Algebraic Groups and Related Structures 52 (2000), 1-60.

[23] Ott-Heinrich Keller, Vorlesungen über algebraische Geometrie, Akademische Verlagsgesellschaft Geest \& Portig K.-G., Leipzig, 1974. MR0429870 (55 \#2880)

[24] János Kollár, Polynomials with integral coefficients, equivalent to a given polynomial, Electron. Res. Announc. Amer. Math. Soc. 3 (1997), 17-27 (electronic), DOI 10.1090/S1079-676297-00019-X. MR1445631 (98g:11076)

[25] A. K. Lenstra, H. W. Lenstra Jr., and L. Lovász, Factoring polynomials with rational coefficients, Math. Ann. 261 (1982), no. 4, 515-534, DOI 10.1007/BF01457454. MR682664 (84a:12002)

[26] Yu. I. Manin, Cubic forms: algebra, geometry, arithmetic, North-Holland Publishing Co., Amsterdam, 1974. Translated from the Russian by M. Hazewinkel; North-Holland Mathematical Library, Vol. 4. MR0460349 (57 \#343)

[27] David Mumford, Lectures on curves on an algebraic surface, With a section by G. M. Bergman. Annals of Mathematics Studies, No. 59, Princeton University Press, Princeton, N.J., 1966. MR0209285 (35 \#187)

[28] David Mumford, Stability of projective varieties, Enseignement Math. (2) 23 (1977), no. 1-2, 39-110. MR0450272 (56 \#8568)

[29] D. Mumford, J. Fogarty, and F. Kirwan, Geometric invariant theory, 3rd ed., Ergebnisse der Mathematik und ihrer Grenzgebiete (2) [Results in Mathematics and Related Areas (2)], vol. 34, Springer-Verlag, Berlin, 1994. MR1304906 (95m:14012)

[30] Isao Naruki, Cross ratio variety as a moduli space of cubic surfaces, Proc. London Math. Soc. (3) 45 (1982), no. 1, 1-30, DOI 10.1112/plms/s3-45.1.1. With an appendix by Eduard Looijenga. MR662660 (84d:14020)

[31] George Salmon, A treatise on the analytic geometry of three dimensions, Revised by R. A. P. Rogers. 7th ed. Vol. 1, Edited by C. H. Rowe. Chelsea Publishing Company, New York, 1958. MR0094753(20 \#1265)

[32] Jean-Pierre Serre, Groupes algébriques et corps de classes (French), Publications de l'institut de mathématique de l'université de Nancago, VII. Hermann, Paris, 1959. MR0103191 (21 \#1973)

[33] Jean-Pierre Serre, Topics in Galois theory, Research Notes in Mathematics, vol. 1, Jones and Bartlett Publishers, Boston, MA, 1992. Lecture notes prepared by Henri Damon [Henri Darmon]; With a foreword by Darmon and the author. MR.1162313 (94d:12006) 
[34] C. P. Sousley, Invariants and Covariants of the Cremona Cubic Surface, Amer. J. Math. 39 (1917), no. 2, 135-146, DOI 10.2307/2370532. MR1506315

[35] Richard P. Stauduhar, The determination of Galois groups, Math. Comp. 27 (1973), 981-996. MR.0327712(48 \#6054)

School of Mathematics and Statistics F07, University of Sydney, New South Wales 2006, Sydney, Australia

E-mail address: stephan@maths.usyd.edu.au

URL: http://www.staff.uni-bayreuth.de/ bt270951/

Département Mathematik, Universität Siegen, Walter-Flex-Str. 3, D-57068 Siegen, GERMANY

E-mail address: jahnel@mathematik.uni-siegen.de

$U R L:$ http://www . uni-math.gwdg.de/jahnel 